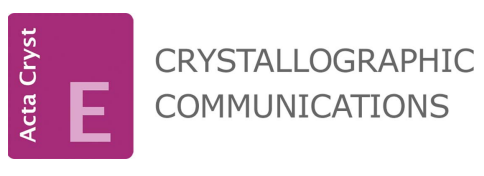

ISSN 2056-9890

Received 21 July 2017

Accepted 26 July 2017

Edited by P. C. Healy, Griffith University, Australia

Keywords: crystal structure; multi-substituted imidazole; hydrogen bonding; $\mathrm{C}-\mathrm{H} \cdots \pi$ interactions.

CCDC reference: 1565059

Supporting information: this article has supporting information at journals.iucr.org/e

\section{Crystal structure of 1-[2-(4-nitrophenyl)-4,5- diphenyl-1H-imidazol-1-yl]propan-2-ol}

\author{
Jim Simpson, ${ }^{\text {a }}$ Shaaban K. Mohamed, ${ }^{\mathrm{b}, \mathrm{c}}$ Adel A. Marzouk, ${ }^{\mathrm{d}}$ Antar A. Abdelhamid \\ and Mustafa R. Albayati ${ }^{f_{*}}$

\begin{abstract}
${ }^{\mathbf{a}}$ Department of Chemistry, University of Otago, PO Box 56, Dunedin, New Zealand, ${ }^{\mathbf{b}}$ Faculty of Science and Engineering, Health Care Division, Manchester Metropolitan University, Manchester M1 5GD, England, ${ }^{\mathbf{c} C h e m i s t r y}$ Department, Faculty of Science, Minia University, 61519 El-Minia, Egypt, dPharmaceutical Chemistry Department, Faculty of Pharmacy, Al Azhar University, 71515 Assiut, Egypt, ${ }^{\mathbf{e} C h e m i s t r y ~ D e p a r t m e n t, ~ F a c u l t y ~ o f ~ S c i e n c e, ~ S o h a g ~}$ University, Sohag, Egypt, and ${ }^{\mathfrak{f}}$ Kirkuk University, College of Education, Department of Chemistry, Kirkuk, Iraq. ${ }^{*}$ Correspondence e-mail: shaabankamel@yahoo.com
\end{abstract}

The title compound, $\mathrm{C}_{24} \mathrm{H}_{21} \mathrm{~N}_{3} \mathrm{O}_{3}$, crystallizes with two unique but closely r.m.s. overlay fit $=0.215 \AA$ ) comparable molecules $(1$ and 2$)$ in the asymmetric unit of the triclinic unit cell. In molecule 1, the dihedral angles between the central imidazlole ring and the benzene-ring substituents are 42.51 (9), 45.41 (9) and $56.92(8)^{\circ}$, respectively. Comparable data for molecule 2 are $39.36(10)$, $34.45(11)$ and $60.34(8)^{\circ}$, respectively. The rings at the 2-positions carry $p$-nitro substituents that subtend dihedral angles of $12.9(4)^{\circ}$ in molecule 1 and $11.7(4)^{\circ}$ in molecule 2 to their respective benzene ring planes. The imidazole rings also have propan-2-ol substituents on the 1-N atoms, which adopt extended conformations for the $\mathrm{N}-\mathrm{C}-\mathrm{C}-\mathrm{C}$ chains. In the crystal, classical $\mathrm{O}-\mathrm{H} \cdots \mathrm{N}$ hydrogen bonds combine with $\mathrm{C}-\mathrm{H} \cdots \mathrm{O}, \mathrm{C}-\mathrm{H} \cdots \mathrm{N}$ and $\mathrm{C}-\mathrm{H} \cdots \pi($ ring) hydrogen bonds and stack the molecules along the $a$-axis direction.

\section{Chemical context}

Imidazole compounds form the core of the structures of some well-known components of human organisms including the amino acid histidine, vitamin-B12, a component of the DNA base structure and the purines, histamine and biotin. It is also present in the structure of many natural or synthetic drug molecules, for example cimetidine, azomycin and metronidazole (Kleeman et al., 1999). Imidazole derivatives display an extensive range of biological activities and are thus used as antibacterial (Vijesh et al., 2011; Lu, et al., 2012), anticancer (Yang et al., 2012; Alkahtani et al., 2012), anti-tubercular (Lu, et al., 2012; Lee et al., 2011), analgesic (Kankala et al., 2013; Gaba et al., 2010) and anti-HIV agents (Zhan et al., 2009). As part of an ongoing study of the synthesis of imidazole-based amino aliphatic alcohols, e.g. amino ethanol and amino isopropanol (Akkurt et al., 2015; Mohamed et al., 2013a,b; Jasinski et al., 2015), we report here the synthesis and crystal structure of the title compound.

\section{Structural commentary}

The title compound, (I), crystallizes with two unique molecules, 1 and 2, in the asymmetric unit. In the numbering scheme these molecules are differentiated by leading 1 and 2 digits, respectively, Fig. 1.

The unique molecules form dimers in the asymmetric unit through $\mathrm{O} 212-\mathrm{H} 210 \cdots \mathrm{N} 13$ and $\mathrm{C} 253-\mathrm{H} 253 \cdots \mathrm{O} 13$ hydrogen bonds that enclose $R_{2}^{2}(18)$ rings, Fig. 1 . The two 
molecules are closely similar and an overlay, Fig. 2 (Macrae et al., 2008), shows an r.m.s. deviation of $0.215 \AA$ with relatively minor variations of the inclinations of the various substituents to the central imidazole rings.

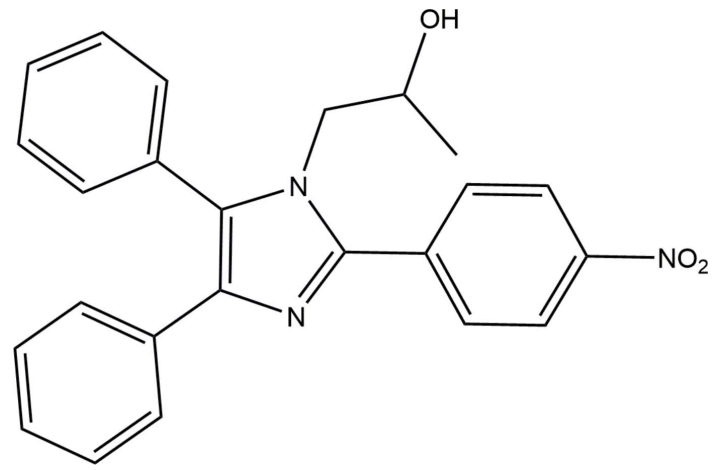

The structure consists of a basic lophine, 2,4,5-triphenyl- $1 H$ imidazole, skeleton (Yanover \& Kaftory, 2009), with isopropanol substituents on the N11 and N21 atoms of the central imidazole rings. The $\mathrm{N}-\mathrm{C}_{3}$ chains of these substituents are planar, with an $\mathrm{N} 1-\mathrm{C} 11-\mathrm{C} 12-\mathrm{C} 13$ torsion angle of $173.09(19)^{\circ}$ in molecule 1 and $171.0(2)^{\circ}$ in molecule 2 ; these planes are inclined to the imidazole ring planes by 74.96 (12) and $74.78(12)^{\circ}$. respectively. The benzene rings are inclined to the imidazole ring plane at angles of 42.51 (9) and $39.36(10)^{\circ}$ for C121-C126 and C221-C226, 45.41 (9) and 34.45 (11) for C141-C146 and C241-C246 and 56.92 (8) and 60.34 (8) for C151-C156 and C251-C256, values that further attest to the close similarities between the structures of the two unique molecules. Bond lengths and angles in the two molecules are

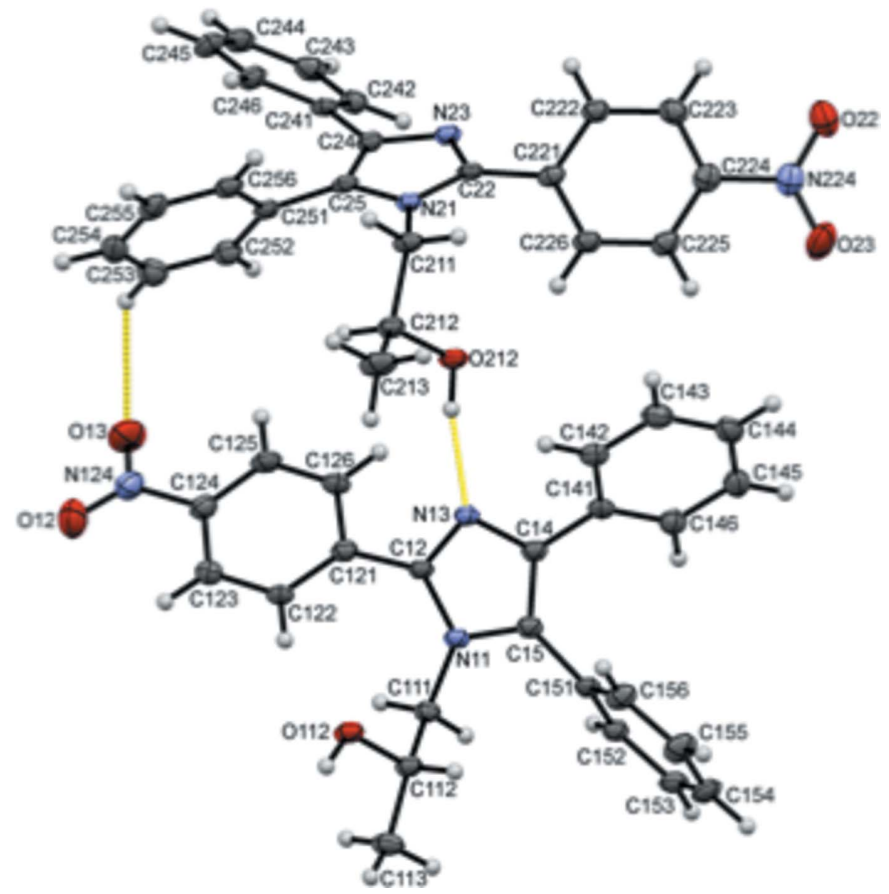

Figure 1

The asymmetric unit of (I), with displacement ellipsoids drawn at the $50 \%$ probability level. Hydrogen bonds between the two unique molecules are shown as yellow dashed lines.
Table 1

Hydrogen-bond geometry $\left(\AA{ }^{\circ}\right)$.

$C g 1$ and $C g 5$ are the centroids of the N11/C12/N13/C14/C15 and N21/C22/ N23/C24/C25 rings, respectively.

\begin{tabular}{lllll}
\hline$D-\mathrm{H} \cdots A$ & $D-\mathrm{H}$ & $\mathrm{H} \cdots A$ & $D \cdots A$ & $D-\mathrm{H} \cdots A$ \\
\hline $\mathrm{O} 212-\mathrm{H} 21 O \cdots \mathrm{N} 13$ & $0.89(4)$ & $1.90(4)$ & $2.773(2)$ & $168(3)$ \\
$\mathrm{C} 253-\mathrm{H} 253 \cdots \mathrm{O} 13$ & 0.95 & 2.69 & $3.491(4)$ & 143 \\
$\mathrm{O} 112-\mathrm{H} 11 O \cdots \mathrm{N} 23^{\mathrm{i}}$ & $0.88(4)$ & $1.94(4)$ & $2.798(2)$ & $165(3)$ \\
$\mathrm{C} 155-\mathrm{H} 155 \cdots \mathrm{O} 22^{\mathrm{i}}$ & 0.95 & 2.57 & $3.244(3)$ & 128 \\
$\mathrm{C} 152-\mathrm{H} 152 \cdots \mathrm{O} 212^{\mathrm{ii}}$ & 0.95 & 2.66 & $3.263(3)$ & 122 \\
$\mathrm{C} 153-\mathrm{H} 153 \cdots \mathrm{N} 21^{\mathrm{ii}}$ & 0.95 & 2.74 & $3.682(3)$ & 170 \\
$\mathrm{C} 243-\mathrm{H} 243 \cdots \mathrm{O} 23^{\mathrm{iii}}$ & 0.95 & 2.59 & $3.542(4)$ & 176 \\
$\mathrm{C} 242-\mathrm{H} 242 \cdots \mathrm{O} 112^{\mathrm{iv}}$ & 0.95 & 2.71 & $3.338(3)$ & 124 \\
$\mathrm{C} 256-\mathrm{H} 256 \cdots \mathrm{O} 112^{\mathrm{v}}$ & 0.95 & 2.57 & $3.208(3)$ & 125 \\
$\mathrm{C} 145-\mathrm{H} 145 \cdots \mathrm{O} 22^{\mathrm{vi}}$ & 0.95 & 2.58 & $3.439(3)$ & 151 \\
$\mathrm{C} 153-\mathrm{H} 153 \cdots C g 5^{\mathrm{ii}}$ & 0.95 & 2.61 & $3.469(2)$ & 151 \\
$\mathrm{C} 255-\mathrm{H} 255 \cdots C g 1^{\mathrm{v}}$ & 0.95 & 2.66 & $3.544(3)$ & 154
\end{tabular}

Symmetry codes: (i) $x, y-1, z$; (ii) $-x+1,-y+1,-z+1$; (iii) $x-1, y, z$; (iv) $x, y+1, z ;(\mathrm{v})-x+1,-y+1,-z+2 ;(\mathrm{vi})-x+2,-y+2,-z+1$.

also similar and compare well with those found in comparable molecules with isopropanol substituents at the 1-position (Jasinski et al., 2015; Mohamed et al., 2017; Akkurt et al., 2015).

\section{Supramolecular features}

In the crystal, classical $\mathrm{O} 112-\mathrm{H} 11 O \cdots \mathrm{N} 23$ and $\mathrm{O} 212-$ $\mathrm{H} 21 O \cdots \mathrm{N} 13$ hydrogen bonds, Table 1 , bolstered by weaker $\mathrm{C} 155-\mathrm{H} 155 \cdots \mathrm{O} 22, \quad \mathrm{C} 242-\mathrm{H} 242 \cdots \mathrm{O} 112$ and $\mathrm{C} 253-$ H253 . O 13 hydrogen bonds link type 1 and type 2 molecules alternately in a head-to-tail fashion into $C(8)$ chains along the $b$-axis direction, Fig. 3. Chains of alternate molecules also form along $c$, in this case head-to-head, due to C153$\mathrm{H} 153 \cdots C g 5$ and $\mathrm{C} 255-\mathrm{H} 255 \cdots C g 1$ contacts $(C g 1$ and $C g 5$ are the centroids of the N11/C12/N13/C14/C15 and N21/C22/

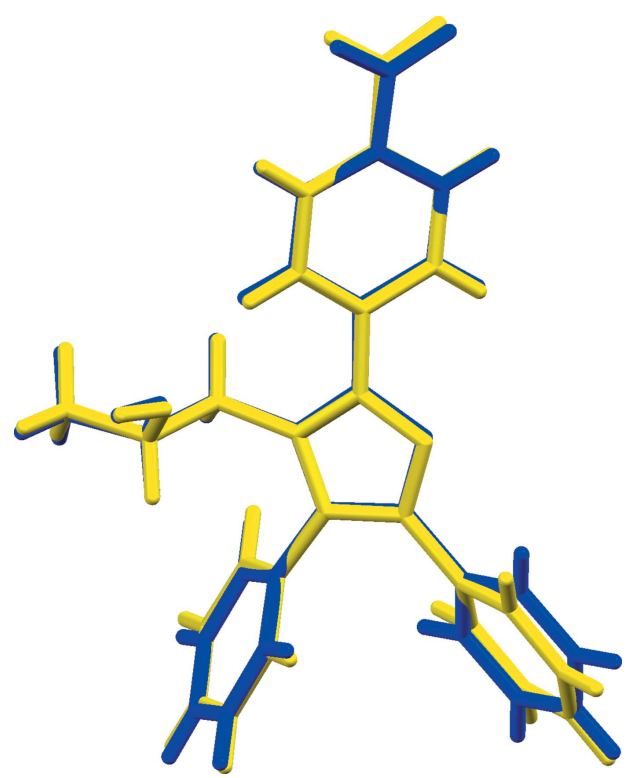

Figure 2

An overlay (Macrae et al., 2008) of the two molecules. Molecule 1 is drawn in yellow with molecule 2 in blue. 


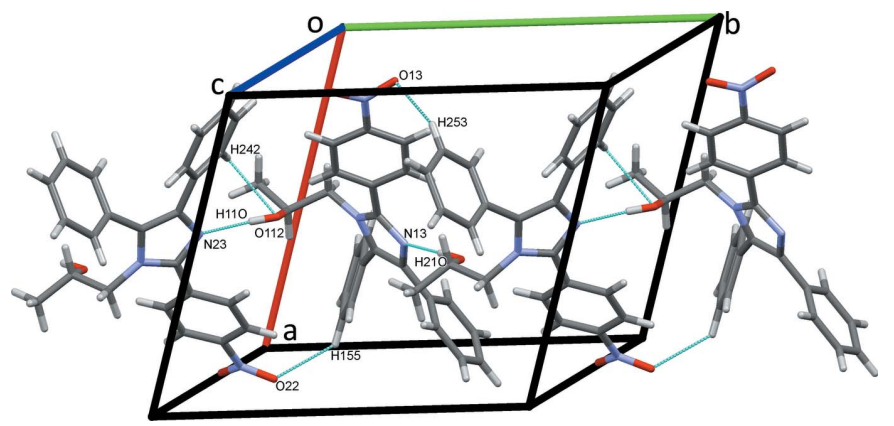

Figure 3

Zigzag chains of molecules of (I) along $b$. In this and subsequent Figures, hydrogen bonds are drawn as dashed lines.

$\mathrm{N} 23 / \mathrm{C} 24 / \mathrm{C} 25$ rings, respectively) combined with $\mathrm{C} 152-$

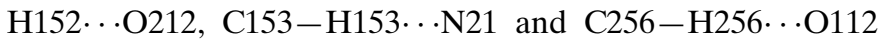
hydrogen bonds, Fig. 4. Chains exclusively of type 2 molecules form along the third axial direction via $\mathrm{C} 243-\mathrm{H} 243 \cdots \mathrm{O} 23$ hydrogen bonds, forming $C(13)$ chains along $a$, Fig. 5. C145$\mathrm{H} 145 \cdots \mathrm{O} 22$ hydrogen bonds link type 1 molecules to these chains, stacking the molecules along $a$. Overall, these numerous contacts generate layers of molecules of (I) stacked along the $a$-axis direction, Fig. 6 .

\section{Database survey}

The Cambridge Structural Database (Version 5.38 with three updates; Groom et al., 2016) shows that molecules with the lophine skeleton and a $\mathrm{CH}_{2}$ substituent on $\mathrm{N} 1$ are reasonably common with 43 entries. However, restricting the search to compounds with isopropanol substituents on $\mathrm{N} 1$ reduces the hits to three reports of our work to produce compounds with 4-benzoic acid (Jasinski et al., 2015) and 4-chloro- (Mohamed et al., 2017) and 2,5-dichloro-substituents (Akkurt et al., 2015) at the 2-position of the imidazole ring. A more recent paper, detailing the use of ionic liquids as catalysts for the preparations of similar compounds, also reports analogues with an unsubstituted phenyl ring and a 2,5-dimethoxy substituted benzene ring at the 2-positions (Marzouk et al. 2017). Other closely related derivatives have ethanol (Mohamed et al., $2013 a$ ) and $n$-propanol substituents on the N1 atom (Mohamed et al., 2015).

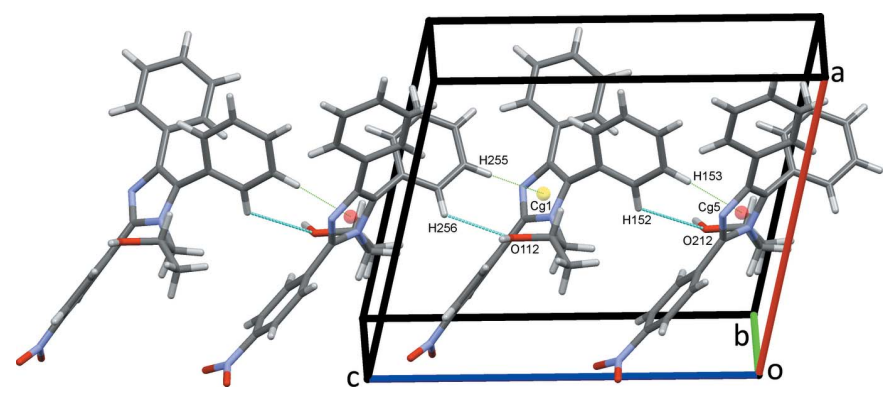

Figure 4

Chains of molecules of (I) along $c$. $\mathrm{C}-\mathrm{H} \cdots \pi$ (ring) contacts are drawn as dotted green lines with ring centroids shown as coloured spheres.

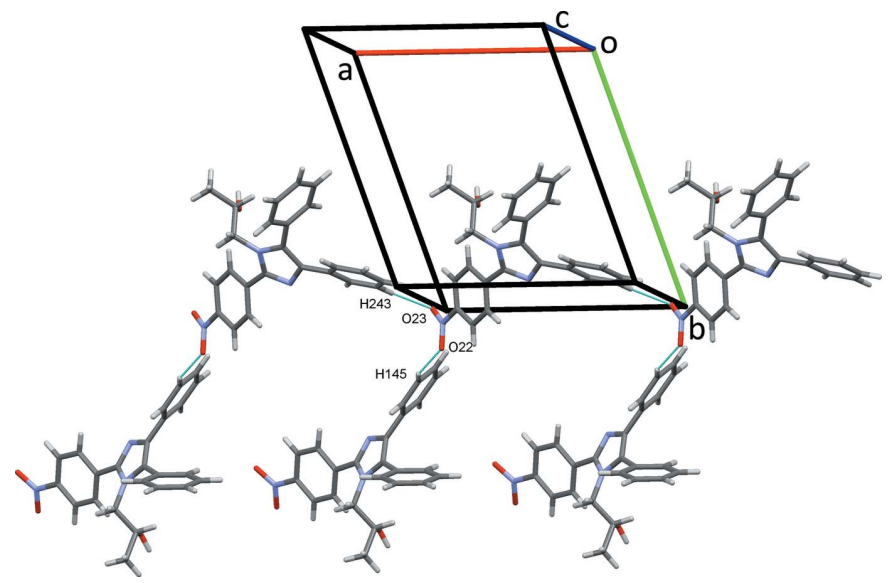

Figure 5

Chains of type 2 molecules of (I) along $a$ linked to type 2 molecules, forming sheets in the $a c$ plane.

\section{Synthesis and crystallization}

The title compound was prepared according to our previously reported method (Marzouk et al., 2017). Crystals suitable for $\mathrm{X}$-ray analysis were obtained by the slow evaporation method using ethanol as a solvent. M.p. 451-453 K, yield, $87 \%$.

\section{Refinement}

Crystal data, data collection and structure refinement details are summarized in Table 2. The hydrogen atoms of the $\mathrm{OH}$ groups on $\mathrm{O} 112$ and $\mathrm{O} 212$ were located in a difference-Fourier map and their coordinates refined with $U_{\text {iso }}=1.5 U_{\text {eq }}(\mathrm{O})$. All other atoms were refined using a riding model with $d(\mathrm{C}-\mathrm{H})=$ $0.95 \AA$ for aromatic, $1.00 \AA$ for methine and $0.98 \AA$ for $\mathrm{CH}_{2}$ atoms, all with $U_{\text {iso }}(\mathrm{H})=1.2 U_{\text {eq }}(\mathrm{C})$. For methyl $\mathrm{H}$ atoms $d(\mathrm{C}-$ $\mathrm{H})=0.98 \AA$ and $U_{\text {iso }}(\mathrm{H})=1.5 U_{\text {eq }}(\mathrm{C})$. One low angle reflection with $F_{\mathrm{o}} \ll F_{\mathrm{c}}$ that may have been affected by the beamstop was omitted from the final refinement cycles.

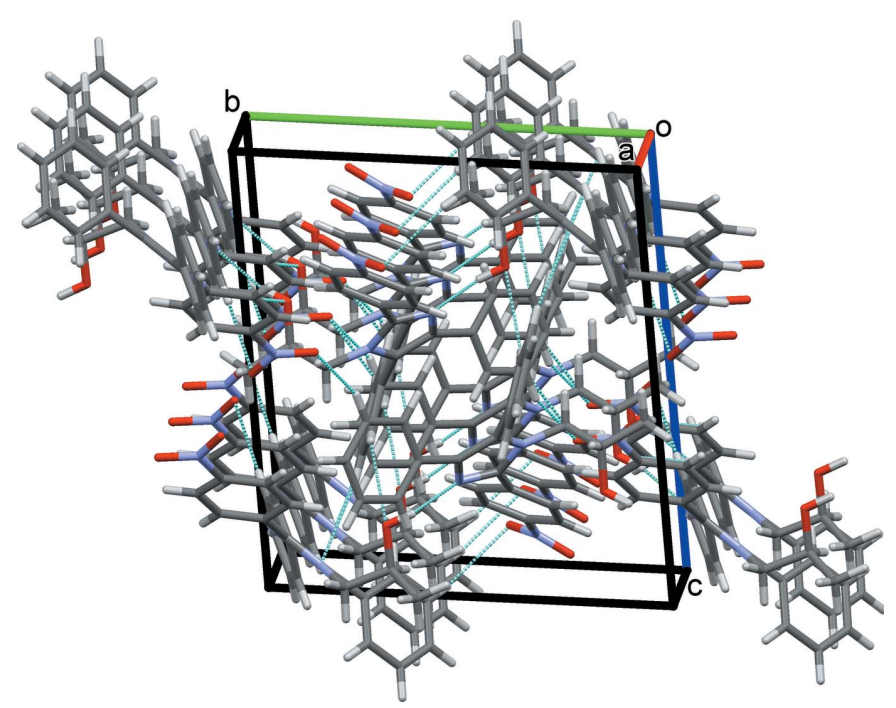

Figure 6

Overall packing of (I) viewed along the $a$-axis direction. 
Table 2

Experimental details.

\begin{tabular}{|c|c|}
\hline \multicolumn{2}{|l|}{ Crystal data } \\
\hline Chemical formula & $\mathrm{C}_{24} \mathrm{H}_{21} \mathrm{~N}_{3} \mathrm{O}_{3}$ \\
\hline$M_{\mathrm{r}}$ & 399.44 \\
\hline Crystal system, space group & Triclinic, $P \overline{1}$ \\
\hline Temperature (K) & 100 \\
\hline$a, b, c(\AA)$ & $\begin{array}{l}12.3070(4), 13.2871(4), \\
13.8499(3)\end{array}$ \\
\hline$\alpha, \beta, \gamma\left(^{\circ}\right)$ & $90.907(2), 100.748(2), 109.938(3)$ \\
\hline$V\left(\AA^{3}\right)$ & 2083.95 (11) \\
\hline$Z$ & 4 \\
\hline Radiation type & $\mathrm{Cu} K \alpha$ \\
\hline$\mu\left(\mathrm{mm}^{-1}\right)$ & 0.69 \\
\hline Crystal size $(\mathrm{mm})$ & $0.52 \times 0.48 \times 0.24$ \\
\hline \multicolumn{2}{|l|}{ Data collection } \\
\hline Diffractometer & $\begin{array}{l}\text { Agilent SuperNova, Dual, } \mathrm{Cu} \text { at } \\
\text { zero, Atlas }\end{array}$ \\
\hline Absorption correction & $\begin{array}{l}\text { Multi-scan (CrysAlis PRO; } \\
\text { Agilent, 2014) }\end{array}$ \\
\hline$T_{\min }, T_{\max }$ & $0.728,1.000$ \\
\hline $\begin{array}{l}\text { No. of measured, independent and } \\
\text { observed }[I>2 \sigma(I)] \text { reflections }\end{array}$ & $42589,8686,7364$ \\
\hline$R_{\text {int }}$ & 0.090 \\
\hline$(\sin \theta / \lambda)_{\max }\left(\AA^{-1}\right)$ & 0.630 \\
\hline \multicolumn{2}{|l|}{ Refinement } \\
\hline$R\left[F^{2}>2 \sigma\left(F^{2}\right)\right], w R\left(F^{2}\right), S$ & $0.072,0.216,1.09$ \\
\hline No. of reflections & 8686 \\
\hline No. of parameters & 549 \\
\hline H-atom treatment & $\begin{array}{l}\mathrm{H} \text { atoms treated by a mixture of } \\
\text { independent and constrained } \\
\text { refinement }\end{array}$ \\
\hline$\Delta \rho_{\max }, \Delta \rho_{\min }\left(\mathrm{e} \AA^{-3}\right)$ & $0.58,-0.39$ \\
\hline
\end{tabular}

Computer programs: CrysAlis PRO (Agilent, 2014), SHELXT (Sheldrick, 2015a), SHELXL2014 (Sheldrick, 2015b) and TITAN (Hunter \& Simpson, 1999), Mercury (Macrae et al., 2008), enCIFer (Allen et al., 2004), PLATON (Spek, 2009), publCIF (Westrip, 2010) and WinGX (Farrugia, 2012).

\section{Acknowledgements}

We thank the University of Otago for the purchase of the diffractometer. We also thank the Department of Chemistry, University of Otago for the support of the work of JS.

\section{References}

Agilent (2014). CrysAlis PRO. Agilent Technologies, Yarnton, England.
Akkurt, M., Jasinski, J. P., Mohamed, S. K., Marzouk, A. A. \& Albayati, M. R. (2015). Acta Cryst. E71, o299-0300.

Alkahtani, H. M., Abbas, A. Y. \& Wang, S. (2012). Bioorg. Med. Chem. Lett. 22, 1317-1321.

Allen, F. H., Johnson, O., Shields, G. P., Smith, B. R. \& Towler, M. (2004). J. Appl. Cryst. 37, 335-338.

Farrugia, L. J. (2012). J. Appl. Cryst. 45, 849-854.

Gaba, M., Singh, D., Singh, S., Sharma, V. \& Gaba, P. (2010). Eur. J. Med. Chem. 45, 2245-2249.

Groom, C. R., Bruno, I. J., Lightfoot, M. P. \& Ward, S. C. (2016). Acta Cryst. B72, 171-179.

Hunter, K. A. \& Simpson, J. (1999). TITAN2000. University of Otago, New Zealand.

Jasinski, J. P., Mohamed, S. K., Akkurt, M., Abdelhamid, A. A. \& Albayati, M. R. (2015). Acta Cryst. E71, o77-o78.

Kankala, S., Kankala, R. K., Gundepaka, P., Thota, N., Nerella, S., Gangula, M. R., Guguloth, H., Kagga, M., Vadde, R. \& Vasam, C. S. (2013). Bioorg. Med. Chem. Lett. 23, 1306-1309.

Kleeman, A., Engel, J., Kutscher, B. \& Reichert, D. (1999). Pharmaceutical Substances, 3rd ed. 2286 pp. Stuttgart/New York: Thieme.

Lee, S., Kim, S., Yun, M., Lee, Y., Cho, S., Oh, T. \& Kim, P. (2011). Bioorg. Med. Chem. Lett. 21, 1515-1518.

Lu, X., Liu, X., Wan, B., Franzblau, S. G., Chen, L., Zhou, C. \& You, Q. (2012). Eur. J. Med. Chem. 49, 164-171.

Macrae, C. F., Bruno, I. J., Chisholm, J. A., Edgington, P. R., McCabe, P., Pidcock, E., Rodriguez-Monge, L., Taylor, R., van de Streek, J. \& Wood, P. A. (2008). J. Appl. Cryst. 41, 466-470.

Marzouk, A. A., Abdelhamid, A. A., Mohamed, S. K. \& Simpson, J. (2017). Z. Naturforsch. Teil B, 72, 23-33.

Mohamed, S. K., Akkurt, M., Marzouk, A. A., Abbasov, V. M. \& Gurbanov, A. V. (2013a). Acta Cryst. E69, o474-o475.

Mohamed, S. K., Akkurt, M., Singh, K., Marzouk, A. A. \& Abdelhamid, A. A. (2013b). Acta Cryst. E69, o1243.

Mohamed, S. K., Marzouk, A. A., Albayati, M. R., Abdelhamid, A. A. \& Simpson, J. (2017). Acta Cryst. E73, 59-62.

Mohamed, S. K., Simpson, J., Marzouk, A. A., Talybov, A. H., Abdelhamid, A. A., Abdullayev, Y. A. \& Abbasov, V. M. (2015). Z. Naturforsch. Teil B, 70, 809-817.

Sheldrick, G. M. (2015a). Acta Cryst. A71, 3-8.

Sheldrick, G. M. (2015b). Acta Cryst. C71, 3-8.

Spek, A. L. (2009). Acta Cryst. D65, 148-155.

Vijesh, A. M., Isloor, A. M., Telkar, S., Peethambar, S. K., Rai, S. \& Isloor, N. (2011). Eur. J. Med. Chem. 46, 3531-3536.

Westrip, S. P. (2010). J. Appl. Cryst. 43, 920-925.

Yang, X., Wan, W., Deng, X., Li, Y., Yang, L., Li, L. \& Zhang, H. (2012). Bioorg. Med. Chem. Lett. 22, 2726-2729.

Yanover, D. \& Kaftory, M. (2009). Acta Cryst. E65, o711.

Zhan, P., Liu, X., Zhu, J., Fang, Z., Li, Z., Pannecouque, C. \& de Clercq, E. (2009). Bioorg. Med. Chem. 17, 5775-5781. 


\section{supporting information}

Acta Cryst. (2017). E73, 1398-1401 [https://doi.org/10.1107/S2056989017011057]

Crystal structure of 1-[2-(4-nitrophenyl)-4,5-diphenyl-1 H-imidazol-1yl]propan-2-ol

Jim Simpson, Shaaban K. Mohamed, Adel A. Marzouk, Antar A. Abdelhamid and Mustafa R. Albayati

Computing details

Data collection: CrysAlis PRO (Agilent, 2014); cell refinement: CrysAlis PRO (Agilent, 2014); data reduction: CrysAlis PRO (Agilent, 2014); program(s) used to solve structure: SHELXT (Sheldrick, 2015a); program(s) used to refine structure: SHELXL2014 (Sheldrick, 2015b) and TITAN (Hunter \& Simpson, 1999); molecular graphics: Mercury (Macrae et al., 2008); software used to prepare material for publication: SHELXL2014 (Sheldrick, 2015b), enCIFer (Allen et al., 2004), PLATON (Spek, 2009), publCIF (Westrip, 2010) and WinGX (Farrugia, 2012).

1-[2-(4-Nitrophenyl)-4,5-diphenyl-1H-imidazol-1-yl] propan-2-ol

Crystal data

$\mathrm{C}_{24} \mathrm{H}_{21} \mathrm{~N}_{3} \mathrm{O}_{3}$

$M_{r}=399.44$

Triclinic, $P \overline{1}$

$a=12.3070(4) \AA$

$b=13.2871$ (4) $\AA$

$c=13.8499$ (3) $\AA$

$\alpha=90.907(2)^{\circ}$

$\beta=100.748(2)^{\circ}$

$\gamma=109.938(3)^{\circ}$

$V=2083.95(11) \AA^{3}$

Data collection

Agilent SuperNova, Dual, $\mathrm{Cu}$ at zero, Atlas diffractometer

Radiation source: SuperNova (Cu) X-ray Source

Detector resolution: 5.1725 pixels $\mathrm{mm}^{-1}$

$\omega$ scans

Absorption correction: multi-scan

(CrysAlis PRO; Agilent, 2014)

$T_{\min }=0.728, T_{\max }=1.000$

Refinement

Refinement on $F^{2}$

Least-squares matrix: full

$R\left[F^{2}>2 \sigma\left(F^{2}\right)\right]=0.072$

$w R\left(F^{2}\right)=0.216$
$Z=4$

$F(000)=840$

$D_{\mathrm{x}}=1.273 \mathrm{Mg} \mathrm{m}^{-3}$

$\mathrm{Cu} K \alpha$ radiation, $\lambda=1.54184 \AA$

Cell parameters from 19318 reflections

$\theta=3.3-76.2^{\circ}$

$\mu=0.69 \mathrm{~mm}^{-1}$

$T=100 \mathrm{~K}$

Block, yellow

$0.52 \times 0.48 \times 0.24 \mathrm{~mm}$

42589 measured reflections

8686 independent reflections

7364 reflections with $I>2 \sigma(I)$

$R_{\text {int }}=0.090$

$\theta_{\text {max }}=76.4^{\circ}, \theta_{\text {min }}=3.3^{\circ}$

$h=-15 \rightarrow 15$

$k=-16 \rightarrow 16$

$l=-17 \rightarrow 17$

$S=1.09$

8686 reflections

549 parameters

0 restraints 
Hydrogen site location: mixed

$\mathrm{H}$ atoms treated by a mixture of independent and constrained refinement

$$
\begin{aligned}
& w=1 /\left[\sigma^{2}\left(F_{\mathrm{o}}^{2}\right)+(0.1211 P)^{2}+1.8979 P\right] \\
& \text { where } P=\left(F_{\mathrm{o}}^{2}+2 F_{\mathrm{c}}^{2}\right) / 3 \\
& (\Delta / \sigma)_{\max }<0.001 \\
& \Delta \rho_{\max }=0.58 \text { e } \AA^{-3} \\
& \Delta \rho_{\min }=-0.39 \mathrm{e} \AA^{-3}
\end{aligned}
$$

Special details

Geometry. All esds (except the esd in the dihedral angle between two 1.s. planes) are estimated using the full covariance matrix. The cell esds are taken into account individually in the estimation of esds in distances, angles and torsion angles; correlations between esds in cell parameters are only used when they are defined by crystal symmetry. An approximate

\begin{tabular}{|c|c|c|c|c|}
\hline & $x$ & $y$ & $z$ & $U_{\text {iso }} * / U_{\text {eq }}$ \\
\hline N11 & $0.47399(16)$ & $0.32171(14)$ & $0.60492(13)$ & $0.0183(4)$ \\
\hline C111 & 0.39745 (19) & $0.21294(16)$ & $0.56313(16)$ & $0.0194(4)$ \\
\hline H11A & 0.3890 & 0.2091 & 0.4905 & $0.023 *$ \\
\hline H11B & 0.3181 & 0.1981 & 0.5779 & $0.023 *$ \\
\hline C112 & $0.4456(2)$ & $0.12685(17)$ & $0.60368(16)$ & $0.0208(4)$ \\
\hline H112 & 0.5289 & 0.1458 & 0.5951 & $0.025 *$ \\
\hline O112 & $0.44400(15)$ & $0.12926(13)$ & $0.70570(11)$ & 0.0224 \\
\hline $\mathrm{H} 11 \mathrm{O}$ & $0.451(3)$ & $0.071(3)$ & $0.731(2)$ & $0.034^{*}$ \\
\hline C113 & $0.3715(2)$ & $0.01767(19)$ & 0.54911 (19) & $0.0293(5)$ \\
\hline $\mathrm{H} 11 \mathrm{C}$ & 0.3980 & -0.0378 & 0.5805 & $0.044^{*}$ \\
\hline H11D & 0.3804 & 0.0183 & 0.4802 & $0.044^{*}$ \\
\hline H11E & 0.2883 & 0.0022 & 0.5514 & $0.044 *$ \\
\hline $\mathrm{C} 12$ & $0.45419(19)$ & $0.38744(16)$ & $0.67156(16)$ & $0.0180(4)$ \\
\hline C121 & $0.35006(19)$ & $0.36587(17)$ & $0.71663(16)$ & $0.0199(4)$ \\
\hline C122 & $0.2969(2)$ & $0.26889(17)$ & $0.75549(17)$ & 0.0218 \\
\hline H122 & 0.3266 & 0.2120 & 0.7520 & $0.026^{*}$ \\
\hline C123 & $0.2005(2)$ & $0.25551(18)$ & $0.79922(18)$ & $0.0247(5)$ \\
\hline H123 & 0.1650 & 0.1903 & 0.8269 & $0.030 *$ \\
\hline $\mathrm{C} 124$ & $0.1573(2)$ & $0.33862(19)$ & $0.80183(18)$ & $0.0259(5)$ \\
\hline N124 & $0.0529(2)$ & $0.32242(18)$ & $0.84456(19)$ & $0.0351(5)$ \\
\hline $\mathrm{O} 12$ & $0.0194(2)$ & 0.24368 (19) & $0.89045(18)$ & $0.0479(6)$ \\
\hline $\mathrm{O} 13$ & $0.00229(19)$ & $0.38771(18)$ & $0.8305(2)$ & $0.0506(6)$ \\
\hline $\mathrm{C} 125$ & $0.2084(2)$ & $0.43608(19)$ & $0.76456(19)$ & $0.0268(5)$ \\
\hline $\mathrm{H} 125$ & 0.1770 & 0.4920 & 0.7670 & $0.032 *$ \\
\hline $\mathrm{C} 126$ & $0.3061(2)$ & $0.44945(18)$ & $0.72384(18)$ & $0.0239(5)$ \\
\hline H126 & 0.3442 & 0.5165 & 0.7003 & $0.029 *$ \\
\hline N13 & $0.54166(16)$ & $0.48177(14)$ & $0.68955(13)$ & $0.0191(4)$ \\
\hline $\mathrm{C} 14$ & $0.62122(19)$ & $0.47744(17)$ & $0.63342(16)$ & $0.0192(4)$ \\
\hline C141 & $0.7291(2)$ & $0.57016(17)$ & $0.63058(17)$ & $0.0212(4)$ \\
\hline C142 & $0.8016(2)$ & $0.62895(18)$ & $0.71690(18)$ & $0.0259(5)$ \\
\hline H142 & 0.7816 & 0.6102 & 0.7788 & $0.031^{*}$ \\
\hline C143 & $0.9035(2)$ & $0.7153(2)$ & $0.7129(2)$ & $0.0315(5)$ \\
\hline
\end{tabular}
(isotropic) treatment of cell esds is used for estimating esds involving l.s. planes.

Refinement. One low angle reflection with $\mathrm{Fo}<<<\mathrm{Fc}$ that may have been affected by the beamstop was omitted from the final refinement cycles.

Fractional atomic coordinates and isotropic or equivalent isotropic displacement parameters $\left(\AA^{2}\right)$ 


\begin{tabular}{|c|c|c|c|c|}
\hline H143 & 0.9533 & 0.7547 & 0.7721 & $0.038^{*}$ \\
\hline C144 & $0.9328(2)$ & 0.74408 (19) & $0.6222(2)$ & $0.0307(5)$ \\
\hline H144 & 1.0024 & 0.8030 & 0.6196 & $0.037 *$ \\
\hline C145 & $0.8599(2)$ & $0.6864(2)$ & $0.5359(2)$ & $0.0297(5)$ \\
\hline H145 & 0.8789 & 0.7061 & 0.4738 & $0.036^{*}$ \\
\hline C146 & $0.7589(2)$ & 0.59978 (19) & $0.54048(18)$ & $0.0250(5)$ \\
\hline H146 & 0.7096 & 0.5601 & 0.4812 & $0.030^{*}$ \\
\hline C15 & 0.58139 (19) & $0.37866(17)$ & $0.58023(16)$ & $0.0192(4)$ \\
\hline C151 & $0.6379(2)$ & $0.33771(17)$ & $0.51106(16)$ & $0.0200(4)$ \\
\hline C152 & $0.5831(2)$ & $0.30527(17)$ & $0.41259(16)$ & $0.0214(4)$ \\
\hline H152 & 0.5049 & 0.3041 & 0.3895 & $0.026^{*}$ \\
\hline C153 & $0.6425(2)$ & $0.27454(18)$ & $0.34782(18)$ & $0.0254(5)$ \\
\hline $\mathrm{H} 153$ & 0.6040 & 0.2512 & 0.2811 & $0.030 *$ \\
\hline C154 & $0.7571(2)$ & $0.2778(2)$ & 0.37998 (19) & $0.0291(5)$ \\
\hline H154 & 0.7975 & 0.2575 & 0.3354 & $0.035^{*}$ \\
\hline $\mathrm{C} 155$ & $0.8127(2)$ & $0.3108(2)$ & $0.4775(2)$ & $0.0308(5)$ \\
\hline H155 & 0.8915 & 0.3134 & 0.4998 & $0.037 *$ \\
\hline C156 & $0.7531(2)$ & 0.34025 (19) & $0.54298(17)$ & $0.0254(5)$ \\
\hline H156 & 0.7915 & 0.3622 & 0.6100 & $0.030^{*}$ \\
\hline $\mathrm{N} 21$ & $0.53514(16)$ & $0.84449(14)$ & $0.89788(13)$ & $0.0178(4)$ \\
\hline C211 & $0.6129(2)$ & $0.78650(17)$ & $0.94029(16)$ & $0.0211(4)$ \\
\hline $\mathrm{H} 21 \mathrm{~A}$ & 0.6196 & 0.7890 & 1.0127 & $0.025^{*}$ \\
\hline $\mathrm{H} 21 \mathrm{~B}$ & 0.6928 & 0.8231 & 0.9271 & $0.025^{*}$ \\
\hline $\mathrm{C} 212$ & $0.5678(2)$ & $0.66925(18)$ & $0.89808(16)$ & $0.0218(4)$ \\
\hline H212 & 0.4833 & 0.6350 & 0.9034 & $0.026^{*}$ \\
\hline $\mathrm{O} 212$ & $0.57451(15)$ & $0.67128(13)$ & $0.79728(12)$ & 0.0247 (4) \\
\hline $\mathrm{H} 21 \mathrm{O}$ & $0.559(3)$ & $0.606(3)$ & $0.769(3)$ & $0.037^{*}$ \\
\hline $\mathrm{C} 213$ & $0.6397(3)$ & $0.6084(2)$ & $0.9554(2)$ & $0.0331(6)$ \\
\hline $\mathrm{H} 21 \mathrm{C}$ & 0.6128 & 0.5346 & 0.9252 & $0.050 *$ \\
\hline $\mathrm{H} 21 \mathrm{D}$ & 0.6294 & 0.6073 & 1.0239 & $0.050^{*}$ \\
\hline $\mathrm{H} 21 \mathrm{E}$ & 0.7234 & 0.6442 & 0.9540 & $0.050 *$ \\
\hline $\mathrm{C} 22$ & $0.55613(19)$ & $0.92431(16)$ & $0.83472(16)$ & $0.0186(4)$ \\
\hline $\mathrm{C} 221$ & 0.65812 (19) & $0.96701(17)$ & $0.78737(16)$ & 0.0193 (4) \\
\hline $\mathrm{C} 222$ & $0.6966(2)$ & $1.07800(18)$ & $0.77616(17)$ & $0.0220(4)$ \\
\hline $\mathrm{H} 222$ & 0.6612 & 1.1216 & 0.8040 & $0.026^{*}$ \\
\hline $\mathrm{C} 223$ & $0.7853(2)$ & $1.12438(19)$ & $0.72527(18)$ & $0.0242(5)$ \\
\hline $\mathrm{H} 223$ & 0.8119 & 1.1994 & 0.7184 & $0.029 *$ \\
\hline C224 & $0.8346(2)$ & 1.05887 (19) & $0.68452(17)$ & $0.0236(5)$ \\
\hline N224 & $0.92519(18)$ & $1.10743(18)$ & $0.62720(16)$ & $0.0287(4)$ \\
\hline $\mathrm{O} 22$ & $0.96751(17)$ & $1.20609(16)$ & $0.63188(15)$ & $0.0366(4)$ \\
\hline $\mathrm{O} 23$ & $0.9549(2)$ & $1.04823(19)$ & $0.57759(18)$ & $0.0479(6)$ \\
\hline $\mathrm{C} 225$ & $0.7994(2)$ & $0.94926(19)$ & $0.69411(17)$ & $0.0235(5)$ \\
\hline $\mathrm{H} 225$ & 0.8348 & 0.9063 & 0.6653 & $0.028 *$ \\
\hline $\mathrm{C} 226$ & $0.7115(2)$ & $0.90354(18)$ & $0.74671(17)$ & $0.0224(4)$ \\
\hline $\mathrm{H} 226$ & 0.6874 & 0.8288 & 0.7551 & $0.027 *$ \\
\hline $\mathrm{N} 23$ & $0.46808(16)$ & $0.96207(14)$ & $0.81808(14)$ & $0.0192(4)$ \\
\hline $\mathrm{C} 24$ & $0.38696(19)$ & $0.90515(17)$ & $0.87159(16)$ & $0.0198(4)$ \\
\hline C241 & 0.27705 (19) & $0.92635(17)$ & $0.87218(17)$ & $0.0203(4)$ \\
\hline
\end{tabular}




$\begin{array}{lllll}\mathrm{C} 242 & 0.2189(2) & 0.95697(18) & 0.78775(17) & 0.0225(4) \\ \mathrm{H} 242 & 0.2500 & 0.9640 & 0.7294 & 0.027^{*} \\ \mathrm{C} 243 & 0.1155(2) & 0.97731(18) & 0.7888(2) & 0.0265(5) \\ \mathrm{H} 243 & 0.0764 & 0.9980 & 0.7309 & 0.032^{*} \\ \mathrm{C} 244 & 0.0689(2) & 0.96790(19) & 0.8728(2) & 0.0299(5) \\ \mathrm{H} 244 & -0.0018 & 0.9819 & 0.8728 & 0.036^{*} \\ \mathrm{C} 245 & 0.1262(2) & 0.9377(2) & 0.9576(2) & 0.0298(5) \\ \mathrm{H} 245 & 0.0946 & 0.9310 & 1.0157 & 0.036^{*} \\ \mathrm{C} 246 & 0.2299(2) & 0.91744(19) & 0.95732(18) & 0.0258(5) \\ \mathrm{H} 246 & 0.2690 & 0.8974 & 1.0155 & 0.031^{*} \\ \mathrm{C} 25 & 0.42676(19) & 0.83105(17) & 0.92131(16) & 0.0188(4) \\ \mathrm{C} 251 & 0.3663(2) & 0.74902(17) & 0.98272(16) & 0.0197(4) \\ \mathrm{C} 252 & 0.2548(2) & 0.67459(19) & 0.94172(18) & 0.0255(5) \\ \mathrm{H} 252 & 0.2227 & 0.6737 & 0.8737 & 0.031^{*} \\ \mathrm{C} 253 & 0.1905(2) & 0.6023(2) & 0.9986(2) & 0.0310(5) \\ \mathrm{H} 253 & 0.1147 & 0.5522 & 0.9697 & 0.037^{*} \\ \mathrm{C} 254 & 0.2371(2) & 0.6029(2) & 1.0986(2) & 0.0306(5) \\ \mathrm{H} 254 & 0.1930 & 0.5537 & 1.1381 & 0.037^{*} \\ \mathrm{C} 255 & 0.3483(2) & 0.6756(2) & 1.13974(18) & 0.0271(5) \\ \mathrm{H} 255 & 0.3807 & 0.6755 & 1.2076 & 0.032^{*} \\ \mathrm{C} 256 & 0.4129(2) & 0.74873(18) & 1.08274(17) & 0.0226(4) \\ \mathrm{H} 256 & 0.4888 & 0.7986 & 1.1118 & 0.027^{*}\end{array}$

Atomic displacement parameters $\left(\AA^{2}\right)$

\begin{tabular}{lllllll}
\hline & $U^{11}$ & $U^{22}$ & $U^{33}$ & $U^{12}$ & $U^{13}$ & $U^{23}$ \\
\hline $\mathrm{N} 11$ & $0.0253(9)$ & $0.0140(8)$ & $0.0201(9)$ & $0.0118(7)$ & $0.0059(7)$ & $0.0034(7)$ \\
$\mathrm{C} 111$ & $0.0244(10)$ & $0.0148(9)$ & $0.0207(10)$ & $0.0093(8)$ & $0.0038(8)$ & $0.0015(8)$ \\
$\mathrm{C} 112$ & $0.0305(11)$ & $0.0165(10)$ & $0.0200(10)$ & $0.0135(8)$ & $0.0061(8)$ & $0.0021(8)$ \\
$\mathrm{O} 112$ & $0.0357(9)$ & $0.0183(7)$ & $0.0199(8)$ & $0.0173(7)$ & $0.0061(6)$ & $0.0033(6)$ \\
$\mathrm{C} 113$ & $0.0416(13)$ & $0.0169(11)$ & $0.0304(12)$ & $0.0128(10)$ & $0.0050(10)$ & $-0.0001(9)$ \\
$\mathrm{C} 12$ & $0.0248(10)$ & $0.0135(9)$ & $0.0193(10)$ & $0.0109(8)$ & $0.0052(8)$ & $0.0018(7)$ \\
$\mathrm{C} 121$ & $0.0233(10)$ & $0.0173(10)$ & $0.0218(10)$ & $0.0106(8)$ & $0.0048(8)$ & $0.0005(8)$ \\
$\mathrm{C} 122$ & $0.0272(11)$ & $0.0151(10)$ & $0.0270(11)$ & $0.0113(8)$ & $0.0080(9)$ & $0.0017(8)$ \\
$\mathrm{C} 123$ & $0.0284(11)$ & $0.0194(10)$ & $0.0277(11)$ & $0.0082(9)$ & $0.0091(9)$ & $0.0015(9)$ \\
$\mathrm{C} 124$ & $0.0248(11)$ & $0.0242(11)$ & $0.0312(12)$ & $0.0099(9)$ & $0.0101(9)$ & $-0.0026(9)$ \\
$\mathrm{N} 124$ & $0.0310(11)$ & $0.0307(11)$ & $0.0463(13)$ & $0.0090(9)$ & $0.0183(10)$ & $-0.0043(10)$ \\
$\mathrm{O} 12$ & $0.0452(12)$ & $0.0458(12)$ & $0.0606(14)$ & $0.0141(10)$ & $0.0324(11)$ & $0.0132(10)$ \\
$\mathrm{O} 13$ & $0.0404(11)$ & $0.0397(12)$ & $0.0850(18)$ & $0.0215(9)$ & $0.0314(11)$ & $0.0010(11)$ \\
$\mathrm{C} 125$ & $0.0303(12)$ & $0.0203(11)$ & $0.0356(13)$ & $0.0145(9)$ & $0.0104(10)$ & $-0.0013(9)$ \\
$\mathrm{C} 126$ & $0.0305(11)$ & $0.0164(10)$ & $0.0288(11)$ & $0.0118(9)$ & $0.0088(9)$ & $0.0025(8)$ \\
$\mathrm{N} 13$ & $0.0259(9)$ & $0.0143(8)$ & $0.0212(9)$ & $0.0107(7)$ & $0.0080(7)$ & $0.0025(7)$ \\
$\mathrm{C} 14$ & $0.0257(10)$ & $0.0165(10)$ & $0.0198(10)$ & $0.0123(8)$ & $0.0060(8)$ & $0.0031(8)$ \\
$\mathrm{C} 141$ & $0.0259(10)$ & $0.0155(10)$ & $0.0277(11)$ & $0.0123(8)$ & $0.0087(9)$ & $0.0036(8)$ \\
$\mathrm{C} 142$ & $0.0323(12)$ & $0.0196(11)$ & $0.0281(12)$ & $0.0108(9)$ & $0.0089(9)$ & $-0.0009(9)$ \\
$\mathrm{C} 143$ & $0.0321(12)$ & $0.0222(11)$ & $0.0398(14)$ & $0.0090(10)$ & $0.0080(11)$ & $-0.0041(10)$ \\
$\mathrm{C} 144$ & $0.0280(12)$ & $0.0204(11)$ & $0.0485(15)$ & $0.0096(9)$ & $0.0172(11)$ & $0.0038(10)$ \\
$\mathrm{C} 145$ & $0.0340(12)$ & $0.0254(12)$ & $0.0382(13)$ & $0.0150(10)$ & $0.0187(11)$ & $0.0098(10)$
\end{tabular}




\begin{tabular}{|c|c|c|c|c|c|c|}
\hline C146 & $0.0308(11)$ & $0.0220(11)$ & $0.0270(11)$ & $0.0129(9)$ & $0.0098(9)$ & $0.0046(9)$ \\
\hline $\mathrm{C} 15$ & $0.0249(10)$ & $0.0163(10)$ & $0.0203(10)$ & $0.0119(8)$ & $0.0051(8)$ & $0.0043(8)$ \\
\hline $\mathrm{C} 151$ & $0.0283(11)$ & $0.0144(9)$ & $0.0234(10)$ & $0.0133(8)$ & $0.0088(9)$ & $0.0048(8)$ \\
\hline $\mathrm{C} 152$ & $0.0275(11)$ & $0.0167(10)$ & $0.0229(11)$ & $0.0110(8)$ & $0.0059(9)$ & $0.0028(8)$ \\
\hline $\mathrm{C} 153$ & $0.0375(12)$ & $0.0181(10)$ & $0.0243(11)$ & $0.0133(9)$ & $0.0087(9)$ & $-0.0004(8)$ \\
\hline $\mathrm{C} 154$ & $0.0421(14)$ & $0.0264(12)$ & $0.0300(12)$ & $0.0218(10)$ & $0.0156(10)$ & $0.0025(9)$ \\
\hline $\mathrm{C} 155$ & $0.0335(12)$ & $0.0344(13)$ & $0.0355(13)$ & $0.0240(11)$ & $0.0101(10)$ & $0.0058(10)$ \\
\hline $\mathrm{C} 156$ & $0.0314(12)$ & $0.0273(11)$ & $0.0237(11)$ & $0.0186(9)$ & $0.0049(9)$ & $0.0040(9)$ \\
\hline N21 & $0.0241(9)$ & $0.0145(8)$ & $0.0189(8)$ & $0.0117(7)$ & $0.0048(7)$ & $0.0007(7)$ \\
\hline $\mathrm{C} 211$ & $0.0283(11)$ & $0.0187(10)$ & $0.0216(10)$ & $0.0163(9)$ & $0.0027(8)$ & $0.0011(8)$ \\
\hline $\mathrm{C} 212$ & $0.0310(11)$ & $0.0184(10)$ & $0.0215(10)$ & $0.0146(9)$ & $0.0067(9)$ & $0.0025(8)$ \\
\hline $\mathrm{O} 212$ & $0.0402(9)$ & $0.0160(7)$ & $0.0231(8)$ & $0.0148(7)$ & $0.0092(7)$ & $0.0009(6)$ \\
\hline $\mathrm{C} 213$ & $0.0511(15)$ & $0.0275(12)$ & $0.0315(13)$ & $0.0284(12)$ & 0.0065 (11) & $0.0046(10)$ \\
\hline $\mathrm{C} 22$ & $0.0260(10)$ & $0.0125(9)$ & $0.0204(10)$ & $0.0108(8)$ & $0.0049(8)$ & $-0.0001(7)$ \\
\hline $\mathrm{C} 221$ & $0.0218(10)$ & $0.0197(10)$ & $0.0199(10)$ & $0.0122(8)$ & $0.0032(8)$ & $0.0010(8)$ \\
\hline $\mathrm{C} 222$ & $0.0260(11)$ & $0.0201(10)$ & $0.0241(11)$ & $0.0132(8)$ & $0.0055(8)$ & $0.0019(8)$ \\
\hline C223 & $0.0265(11)$ & $0.0207(10)$ & $0.0280(11)$ & $0.0115(9)$ & $0.0054(9)$ & 0.0055 (9) \\
\hline $\mathrm{C} 224$ & $0.0228(10)$ & $0.0279(12)$ & $0.0240(11)$ & $0.0137(9)$ & $0.0048(8)$ & $0.0060(9)$ \\
\hline N224 & $0.0281(10)$ & $0.0347(11)$ & $0.0289(10)$ & $0.0159(9)$ & $0.0093(8)$ & $0.0099(9)$ \\
\hline $\mathrm{O} 22$ & $0.0342(9)$ & $0.0351(10)$ & $0.0410(11)$ & $0.0093(8)$ & $0.0134(8)$ & $0.0129(8)$ \\
\hline $\mathrm{O} 23$ & $0.0586(13)$ & $0.0499(13)$ & $0.0568(14)$ & $0.0314(11)$ & $0.0390(11)$ & $0.0159(11)$ \\
\hline $\mathrm{C} 225$ & $0.0264(11)$ & $0.0251(11)$ & $0.0249(11)$ & $0.0160(9)$ & $0.0064(9)$ & $0.0028(9)$ \\
\hline $\mathrm{C} 226$ & $0.0278(11)$ & $0.0190(10)$ & $0.0250(11)$ & $0.0141(9)$ & $0.0055(9)$ & $0.0022(8)$ \\
\hline N23 & $0.0245(9)$ & $0.0161(8)$ & $0.0221(9)$ & $0.0129(7)$ & $0.0061(7)$ & $0.0017(7)$ \\
\hline $\mathrm{C} 24$ & $0.0257(10)$ & $0.0181(10)$ & $0.0197(10)$ & $0.0125(8)$ & $0.0055(8)$ & $0.0001(8)$ \\
\hline $\mathrm{C} 241$ & $0.0248(10)$ & $0.0141(9)$ & $0.0260(11)$ & $0.0112(8)$ & $0.0070(8)$ & $-0.0004(8)$ \\
\hline $\mathrm{C} 242$ & $0.0240(10)$ & $0.0191(10)$ & $0.0275(11)$ & $0.0107(8)$ & $0.0065(9)$ & $0.0033(8)$ \\
\hline $\mathrm{C} 243$ & $0.0244(11)$ & $0.0196(10)$ & $0.0388(13)$ & $0.0126(9)$ & $0.0046(9)$ & $0.0032(9)$ \\
\hline $\mathrm{C} 244$ & $0.0261(11)$ & $0.0218(11)$ & $0.0464(15)$ & $0.0127(9)$ & $0.0110(10)$ & $-0.0016(10)$ \\
\hline $\mathrm{C} 245$ & $0.0348(13)$ & $0.0279(12)$ & $0.0353(13)$ & $0.0172(10)$ & $0.0164(10)$ & $-0.0003(10)$ \\
\hline $\mathrm{C} 246$ & $0.0339(12)$ & $0.0241(11)$ & $0.0266(11)$ & $0.0175(9)$ & $0.0098(9)$ & $0.0015(9)$ \\
\hline $\mathrm{C} 25$ & $0.0253(10)$ & $0.0162(9)$ & $0.0188(10)$ & $0.0123(8)$ & $0.0041(8)$ & $-0.0021(8)$ \\
\hline C251 & $0.0286(11)$ & $0.0165(10)$ & $0.0204(10)$ & $0.0144(8)$ & $0.0082(8)$ & $0.0019(8)$ \\
\hline $\mathrm{C} 252$ & $0.0301(11)$ & $0.0233(11)$ & $0.0257(11)$ & $0.0128(9)$ & $0.0057(9)$ & $0.0001(9)$ \\
\hline $\mathrm{C} 253$ & $0.0321(12)$ & $0.0240(12)$ & $0.0392(14)$ & $0.0112(10)$ & $0.0104(10)$ & $0.0030(10)$ \\
\hline $\mathrm{C} 254$ & $0.0412(13)$ & $0.0238(11)$ & $0.0394(14)$ & $0.0201(10)$ & $0.0211(11)$ & $0.0133(10)$ \\
\hline $\mathrm{C} 255$ & $0.0420(13)$ & $0.0282(12)$ & $0.0244(11)$ & $0.0255(10)$ & $0.0136(10)$ & $0.0095(9)$ \\
\hline $\mathrm{C} 256$ & $0.0319(11)$ & $0.0205(10)$ & $0.0225(11)$ & $0.0176(9)$ & $0.0066(9)$ & $0.0018(8)$ \\
\hline
\end{tabular}

Geometric parameters $\left(\AA,{ }^{\circ}\right)$

\begin{tabular}{llll}
\hline $\mathrm{N} 11-\mathrm{C} 12$ & $1.370(3)$ & $\mathrm{N} 21-\mathrm{C} 22$ & $1.372(3)$ \\
$\mathrm{N} 11-\mathrm{C} 15$ & $1.390(3)$ & $\mathrm{N} 21-\mathrm{C} 25$ & $1.385(3)$ \\
$\mathrm{N} 11-\mathrm{C} 111$ & $1.467(3)$ & $\mathrm{N} 21-\mathrm{C} 211$ & $1.471(3)$ \\
$\mathrm{C} 111-\mathrm{C} 112$ & $1.527(3)$ & $\mathrm{C} 211-\mathrm{C} 212$ & $1.529(3)$ \\
$\mathrm{C} 111-\mathrm{H} 11 \mathrm{~A}$ & 0.9900 & $\mathrm{C} 211-\mathrm{H} 21 \mathrm{~A}$ & 0.9900 \\
$\mathrm{C} 111-\mathrm{H} 11 \mathrm{~B}$ & 0.9900 & $\mathrm{C} 211-\mathrm{H} 21 \mathrm{~B}$ & 0.9900 \\
$\mathrm{C} 112-\mathrm{O} 112$ & $1.417(3)$ & $\mathrm{C} 212-\mathrm{O} 212$ & $1.414(3)$
\end{tabular}




\begin{tabular}{|c|c|c|c|}
\hline $\mathrm{C} 112-\mathrm{C} 113$ & $1.521(3)$ & $\mathrm{C} 212-\mathrm{C} 213$ & $1.521(3)$ \\
\hline C112-H112 & 1.0000 & $\mathrm{C} 212-\mathrm{H} 212$ & 1.0000 \\
\hline $\mathrm{O} 112-\mathrm{H} 11 \mathrm{O}$ & $0.88(4)$ & $\mathrm{O} 212-\mathrm{H} 21 \mathrm{O}$ & $0.89(4)$ \\
\hline $\mathrm{C} 113-\mathrm{H} 11 \mathrm{C}$ & 0.9800 & $\mathrm{C} 213-\mathrm{H} 21 \mathrm{C}$ & 0.9800 \\
\hline C113-H11D & 0.9800 & $\mathrm{C} 213-\mathrm{H} 21 \mathrm{D}$ & 0.9800 \\
\hline C113-H11E & 0.9800 & $\mathrm{C} 213-\mathrm{H} 21 \mathrm{E}$ & 0.9800 \\
\hline $\mathrm{C} 12-\mathrm{N} 13$ & $1.327(3)$ & $\mathrm{C} 22-\mathrm{N} 23$ & $1.327(3)$ \\
\hline $\mathrm{C} 12-\mathrm{C} 121$ & $1.472(3)$ & $\mathrm{C} 22-\mathrm{C} 221$ & $1.470(3)$ \\
\hline $\mathrm{C} 121-\mathrm{C} 122$ & $1.399(3)$ & $\mathrm{C} 221-\mathrm{C} 226$ & $1.400(3)$ \\
\hline $\mathrm{C} 121-\mathrm{C} 126$ & $1.401(3)$ & $\mathrm{C} 221-\mathrm{C} 222$ & $1.408(3)$ \\
\hline $\mathrm{C} 122-\mathrm{C} 123$ & $1.389(3)$ & $\mathrm{C} 222-\mathrm{C} 223$ & $1.382(3)$ \\
\hline C122-H122 & 0.9500 & $\mathrm{C} 222-\mathrm{H} 222$ & 0.9500 \\
\hline $\mathrm{C} 123-\mathrm{C} 124$ & $1.382(3)$ & $\mathrm{C} 223-\mathrm{C} 224$ & $1.384(3)$ \\
\hline C123-H123 & 0.9500 & $\mathrm{C} 223-\mathrm{H} 223$ & 0.9500 \\
\hline $\mathrm{C} 124-\mathrm{C} 125$ & $1.388(3)$ & $\mathrm{C} 224-\mathrm{C} 225$ & $1.387(3)$ \\
\hline $\mathrm{C} 124-\mathrm{N} 124$ & $1.465(3)$ & $\mathrm{C} 224-\mathrm{N} 224$ & $1.465(3)$ \\
\hline $\mathrm{N} 124-\mathrm{O} 12$ & $1.222(3)$ & $\mathrm{N} 224-\mathrm{O} 23$ & $1.224(3)$ \\
\hline $\mathrm{N} 124-\mathrm{O} 13$ & $1.228(3)$ & $\mathrm{N} 224-\mathrm{O} 22$ & $1.230(3)$ \\
\hline $\mathrm{C} 125-\mathrm{C} 126$ & $1.381(3)$ & $\mathrm{C} 225-\mathrm{C} 226$ & $1.390(3)$ \\
\hline $\mathrm{C} 125-\mathrm{H} 125$ & 0.9500 & $\mathrm{C} 225-\mathrm{H} 225$ & 0.9500 \\
\hline $\mathrm{C} 126-\mathrm{H} 126$ & 0.9500 & $\mathrm{C} 226-\mathrm{H} 226$ & 0.9500 \\
\hline $\mathrm{N} 13-\mathrm{C} 14$ & $1.374(3)$ & $\mathrm{N} 23-\mathrm{C} 24$ & $1.377(3)$ \\
\hline $\mathrm{C} 14-\mathrm{C} 15$ & $1.379(3)$ & $\mathrm{C} 24-\mathrm{C} 25$ & $1.381(3)$ \\
\hline $\mathrm{C} 14-\mathrm{C} 141$ & $1.479(3)$ & $\mathrm{C} 24-\mathrm{C} 241$ & $1.474(3)$ \\
\hline $\mathrm{C} 141-\mathrm{C} 142$ & $1.391(3)$ & $\mathrm{C} 241-\mathrm{C} 242$ & $1.395(3)$ \\
\hline $\mathrm{C} 141-\mathrm{C} 146$ & $1.391(3)$ & $\mathrm{C} 241-\mathrm{C} 246$ & $1.398(3)$ \\
\hline $\mathrm{C} 142-\mathrm{C} 143$ & $1.392(3)$ & $\mathrm{C} 242-\mathrm{C} 243$ & $1.391(3)$ \\
\hline $\mathrm{C} 142-\mathrm{H} 142$ & 0.9500 & $\mathrm{C} 242-\mathrm{H} 242$ & 0.9500 \\
\hline $\mathrm{C} 143-\mathrm{C} 144$ & $1.394(4)$ & $\mathrm{C} 243-\mathrm{C} 244$ & $1.380(4)$ \\
\hline C143-H143 & 0.9500 & $\mathrm{C} 243-\mathrm{H} 243$ & 0.9500 \\
\hline $\mathrm{C} 144-\mathrm{C} 145$ & $1.387(4)$ & $\mathrm{C} 244-\mathrm{C} 245$ & $1.391(4)$ \\
\hline C144-H144 & 0.9500 & $\mathrm{C} 244-\mathrm{H} 244$ & 0.9500 \\
\hline $\mathrm{C} 145-\mathrm{C} 146$ & $1.389(3)$ & $\mathrm{C} 245-\mathrm{C} 246$ & $1.392(3)$ \\
\hline $\mathrm{C} 145-\mathrm{H} 145$ & 0.9500 & $\mathrm{C} 245-\mathrm{H} 245$ & 0.9500 \\
\hline C146-H146 & 0.9500 & $\mathrm{C} 246-\mathrm{H} 246$ & 0.9500 \\
\hline $\mathrm{C} 15-\mathrm{C} 151$ & $1.479(3)$ & $\mathrm{C} 25-\mathrm{C} 251$ & $1.475(3)$ \\
\hline $\mathrm{C} 151-\mathrm{C} 152$ & $1.393(3)$ & $\mathrm{C} 251-\mathrm{C} 252$ & $1.397(3)$ \\
\hline $\mathrm{C} 151-\mathrm{C} 156$ & $1.394(3)$ & $\mathrm{C} 251-\mathrm{C} 256$ & $1.398(3)$ \\
\hline $\mathrm{C} 152-\mathrm{C} 153$ & $1.394(3)$ & $\mathrm{C} 252-\mathrm{C} 253$ & $1.381(4)$ \\
\hline C152-H152 & 0.9500 & $\mathrm{C} 252-\mathrm{H} 252$ & 0.9500 \\
\hline $\mathrm{C} 153-\mathrm{C} 154$ & $1.383(4)$ & $\mathrm{C} 253-\mathrm{C} 254$ & $1.395(4)$ \\
\hline C153-H153 & 0.9500 & $\mathrm{C} 253-\mathrm{H} 253$ & 0.9500 \\
\hline $\mathrm{C} 154-\mathrm{C} 155$ & $1.384(4)$ & $\mathrm{C} 254-\mathrm{C} 255$ & $1.386(4)$ \\
\hline C154-H154 & 0.9500 & $\mathrm{C} 254-\mathrm{H} 254$ & 0.9500 \\
\hline $\mathrm{C} 155-\mathrm{C} 156$ & $1.395(3)$ & $\mathrm{C} 255-\mathrm{C} 256$ & $1.390(3)$ \\
\hline C155-H155 & 0.9500 & $\mathrm{C} 255-\mathrm{H} 255$ & 0.9500 \\
\hline $\mathrm{C} 156-\mathrm{H} 156$ & 0.9500 & $\mathrm{C} 256-\mathrm{H} 256$ & 0.9500 \\
\hline
\end{tabular}




\begin{tabular}{|c|c|c|c|}
\hline $\mathrm{C} 12-\mathrm{N} 11-\mathrm{C} 15$ & $107.00(18)$ & $\mathrm{C} 22-\mathrm{N} 21-\mathrm{C} 25$ & $107.17(17)$ \\
\hline $\mathrm{C} 12-\mathrm{N} 11-\mathrm{C} 111$ & $128.14(18)$ & $\mathrm{C} 22-\mathrm{N} 21-\mathrm{C} 211$ & $128.44(18)$ \\
\hline $\mathrm{C} 15-\mathrm{N} 11-\mathrm{C} 111$ & $124.83(18)$ & $\mathrm{C} 25-\mathrm{N} 21-\mathrm{C} 211$ & $124.28(18)$ \\
\hline $\mathrm{N} 11-\mathrm{C} 111-\mathrm{C} 112$ & $112.57(17)$ & $\mathrm{N} 21-\mathrm{C} 211-\mathrm{C} 212$ & $112.49(18)$ \\
\hline $\mathrm{N} 11-\mathrm{C} 111-\mathrm{H} 11 \mathrm{~A}$ & 109.1 & $\mathrm{~N} 21-\mathrm{C} 211-\mathrm{H} 21 \mathrm{~A}$ & 109.1 \\
\hline $\mathrm{C} 112-\mathrm{C} 111-\mathrm{H} 11 \mathrm{~A}$ & 109.1 & $\mathrm{C} 212-\mathrm{C} 211-\mathrm{H} 21 \mathrm{~A}$ & 109.1 \\
\hline $\mathrm{N} 11-\mathrm{C} 111-\mathrm{H} 11 \mathrm{~B}$ & 109.1 & $\mathrm{~N} 21-\mathrm{C} 211-\mathrm{H} 21 \mathrm{~B}$ & 109.1 \\
\hline $\mathrm{C} 112-\mathrm{C} 111-\mathrm{H} 11 \mathrm{~B}$ & 109.1 & $\mathrm{C} 212-\mathrm{C} 211-\mathrm{H} 21 \mathrm{~B}$ & 109.1 \\
\hline $\mathrm{H} 11 \mathrm{~A}-\mathrm{C} 111-\mathrm{H} 11 \mathrm{~B}$ & 107.8 & $\mathrm{H} 21 \mathrm{~A}-\mathrm{C} 211-\mathrm{H} 21 \mathrm{~B}$ & 107.8 \\
\hline $\mathrm{O} 112-\mathrm{C} 112-\mathrm{C} 113$ & $112.54(18)$ & $\mathrm{O} 212-\mathrm{C} 212-\mathrm{C} 213$ & $113.03(19)$ \\
\hline $\mathrm{O} 112-\mathrm{C} 112-\mathrm{C} 111$ & $106.18(17)$ & $\mathrm{O} 212-\mathrm{C} 212-\mathrm{C} 211$ & $106.32(17)$ \\
\hline $\mathrm{C} 113-\mathrm{C} 112-\mathrm{C} 111$ & $110.42(18)$ & $\mathrm{C} 213-\mathrm{C} 212-\mathrm{C} 211$ & $110.40(19)$ \\
\hline $\mathrm{O} 112-\mathrm{C} 112-\mathrm{H} 112$ & 109.2 & $\mathrm{O} 212-\mathrm{C} 212-\mathrm{H} 212$ & 109.0 \\
\hline $\mathrm{C} 113-\mathrm{C} 112-\mathrm{H} 112$ & 109.2 & $\mathrm{C} 213-\mathrm{C} 212-\mathrm{H} 212$ & 109.0 \\
\hline $\mathrm{C} 111-\mathrm{C} 112-\mathrm{H} 112$ & 109.2 & $\mathrm{C} 211-\mathrm{C} 212-\mathrm{H} 212$ & 109.0 \\
\hline $\mathrm{C} 112-\mathrm{O} 112-\mathrm{H} 11 \mathrm{O}$ & $111(2)$ & $\mathrm{C} 212-\mathrm{O} 212-\mathrm{H} 21 \mathrm{O}$ & $112(2)$ \\
\hline $\mathrm{C} 112-\mathrm{C} 113-\mathrm{H} 11 \mathrm{C}$ & 109.5 & $\mathrm{C} 212-\mathrm{C} 213-\mathrm{H} 21 \mathrm{C}$ & 109.5 \\
\hline $\mathrm{C} 112-\mathrm{C} 113-\mathrm{H} 11 \mathrm{D}$ & 109.5 & $\mathrm{C} 212-\mathrm{C} 213-\mathrm{H} 21 \mathrm{D}$ & 109.5 \\
\hline $\mathrm{H} 11 \mathrm{C}-\mathrm{C} 113-\mathrm{H} 11 \mathrm{D}$ & 109.5 & $\mathrm{H} 21 \mathrm{C}-\mathrm{C} 213-\mathrm{H} 21 \mathrm{D}$ & 109.5 \\
\hline $\mathrm{C} 112-\mathrm{C} 113-\mathrm{H} 11 \mathrm{E}$ & 109.5 & $\mathrm{C} 212-\mathrm{C} 213-\mathrm{H} 21 \mathrm{E}$ & 109.5 \\
\hline $\mathrm{H} 11 \mathrm{C}-\mathrm{C} 113-\mathrm{H} 11 \mathrm{E}$ & 109.5 & $\mathrm{H} 21 \mathrm{C}-\mathrm{C} 213-\mathrm{H} 21 \mathrm{E}$ & 109.5 \\
\hline $\mathrm{H} 11 \mathrm{D}-\mathrm{C} 113-\mathrm{H} 11 \mathrm{E}$ & 109.5 & $\mathrm{H} 21 \mathrm{D}-\mathrm{C} 213-\mathrm{H} 21 \mathrm{E}$ & 109.5 \\
\hline $\mathrm{N} 13-\mathrm{C} 12-\mathrm{N} 11$ & $110.95(19)$ & $\mathrm{N} 23-\mathrm{C} 22-\mathrm{N} 21$ & $110.80(19)$ \\
\hline $\mathrm{N} 13-\mathrm{C} 12-\mathrm{C} 121$ & $121.03(19)$ & $\mathrm{N} 23-\mathrm{C} 22-\mathrm{C} 221$ & $120.87(19)$ \\
\hline $\mathrm{N} 11-\mathrm{C} 12-\mathrm{C} 121$ & $127.94(19)$ & $\mathrm{N} 21-\mathrm{C} 22-\mathrm{C} 221$ & $128.32(18)$ \\
\hline $\mathrm{C} 122-\mathrm{C} 121-\mathrm{C} 126$ & $119.1(2)$ & $\mathrm{C} 226-\mathrm{C} 221-\mathrm{C} 222$ & $119.1(2)$ \\
\hline $\mathrm{C} 122-\mathrm{C} 121-\mathrm{C} 12$ & $123.53(19)$ & $\mathrm{C} 226-\mathrm{C} 221-\mathrm{C} 22$ & $124.2(2)$ \\
\hline $\mathrm{C} 126-\mathrm{C} 121-\mathrm{C} 12$ & $117.30(19)$ & $\mathrm{C} 222-\mathrm{C} 221-\mathrm{C} 22$ & $116.45(19)$ \\
\hline $\mathrm{C} 123-\mathrm{C} 122-\mathrm{C} 121$ & $120.2(2)$ & $\mathrm{C} 223-\mathrm{C} 222-\mathrm{C} 221$ & $120.9(2)$ \\
\hline $\mathrm{C} 123-\mathrm{C} 122-\mathrm{H} 122$ & 119.9 & $\mathrm{C} 223-\mathrm{C} 222-\mathrm{H} 222$ & 119.5 \\
\hline $\mathrm{C} 121-\mathrm{C} 122-\mathrm{H} 122$ & 119.9 & $\mathrm{C} 221-\mathrm{C} 222-\mathrm{H} 222$ & 119.5 \\
\hline $\mathrm{C} 124-\mathrm{C} 123-\mathrm{C} 122$ & $118.9(2)$ & $\mathrm{C} 222-\mathrm{C} 223-\mathrm{C} 224$ & $118.4(2)$ \\
\hline $\mathrm{C} 124-\mathrm{C} 123-\mathrm{H} 123$ & 120.6 & $\mathrm{C} 222-\mathrm{C} 223-\mathrm{H} 223$ & 120.8 \\
\hline $\mathrm{C} 122-\mathrm{C} 123-\mathrm{H} 123$ & 120.6 & $\mathrm{C} 224-\mathrm{C} 223-\mathrm{H} 223$ & 120.8 \\
\hline $\mathrm{C} 123-\mathrm{C} 124-\mathrm{C} 125$ & $122.4(2)$ & $\mathrm{C} 223-\mathrm{C} 224-\mathrm{C} 225$ & $122.5(2)$ \\
\hline $\mathrm{C} 123-\mathrm{C} 124-\mathrm{N} 124$ & $118.6(2)$ & $\mathrm{C} 223-\mathrm{C} 224-\mathrm{N} 224$ & $118.3(2)$ \\
\hline $\mathrm{C} 125-\mathrm{C} 124-\mathrm{N} 124$ & $119.0(2)$ & $\mathrm{C} 225-\mathrm{C} 224-\mathrm{N} 224$ & $119.2(2)$ \\
\hline $\mathrm{O} 12-\mathrm{N} 124-\mathrm{O} 13$ & $123.5(2)$ & $\mathrm{O} 23-\mathrm{N} 224-\mathrm{O} 22$ & $123.5(2)$ \\
\hline $\mathrm{O} 12-\mathrm{N} 124-\mathrm{C} 124$ & $118.4(2)$ & $\mathrm{O} 23-\mathrm{N} 224-\mathrm{C} 224$ & $118.6(2)$ \\
\hline $\mathrm{O} 13-\mathrm{N} 124-\mathrm{C} 124$ & $118.1(2)$ & $\mathrm{O} 22-\mathrm{N} 224-\mathrm{C} 224$ & $117.8(2)$ \\
\hline $\mathrm{C} 126-\mathrm{C} 125-\mathrm{C} 124$ & $118.1(2)$ & $\mathrm{C} 224-\mathrm{C} 225-\mathrm{C} 226$ & $118.7(2)$ \\
\hline $\mathrm{C} 126-\mathrm{C} 125-\mathrm{H} 125$ & 120.9 & $\mathrm{C} 224-\mathrm{C} 225-\mathrm{H} 225$ & 120.6 \\
\hline $\mathrm{C} 124-\mathrm{C} 125-\mathrm{H} 125$ & 120.9 & $\mathrm{C} 226-\mathrm{C} 225-\mathrm{H} 225$ & 120.6 \\
\hline $\mathrm{C} 125-\mathrm{C} 126-\mathrm{C} 121$ & $121.2(2)$ & $\mathrm{C} 225-\mathrm{C} 226-\mathrm{C} 221$ & $120.3(2)$ \\
\hline $\mathrm{C} 125-\mathrm{C} 126-\mathrm{H} 126$ & 119.4 & $\mathrm{C} 225-\mathrm{C} 226-\mathrm{H} 226$ & 119.8 \\
\hline $\mathrm{C} 121-\mathrm{C} 126-\mathrm{H} 126$ & 119.4 & $\mathrm{C} 221-\mathrm{C} 226-\mathrm{H} 226$ & 119.8 \\
\hline $\mathrm{C} 12-\mathrm{N} 13-\mathrm{C} 14$ & $106.47(18)$ & $\mathrm{C} 22-\mathrm{N} 23-\mathrm{C} 24$ & $106.57(18)$ \\
\hline $\mathrm{N} 13-\mathrm{C} 14-\mathrm{C} 15$ & $109.86(19)$ & $\mathrm{N} 23-\mathrm{C} 24-\mathrm{C} 25$ & $109.58(19)$ \\
\hline
\end{tabular}




\begin{tabular}{|c|c|c|c|}
\hline $\mathrm{N} 13-\mathrm{C} 14-\mathrm{C} 141$ & $122.40(19)$ & $\mathrm{N} 23-\mathrm{C} 24-\mathrm{C} 241$ & $122.00(19)$ \\
\hline $\mathrm{C} 15-\mathrm{C} 14-\mathrm{C} 141$ & $127.7(2)$ & $\mathrm{C} 25-\mathrm{C} 24-\mathrm{C} 241$ & $128.4(2)$ \\
\hline $\mathrm{C} 142-\mathrm{C} 141-\mathrm{C} 146$ & $119.1(2)$ & $\mathrm{C} 242-\mathrm{C} 241-\mathrm{C} 246$ & $118.8(2)$ \\
\hline $\mathrm{C} 142-\mathrm{C} 141-\mathrm{C} 14$ & $121.0(2)$ & $\mathrm{C} 242-\mathrm{C} 241-\mathrm{C} 24$ & $120.4(2)$ \\
\hline $\mathrm{C} 146-\mathrm{C} 141-\mathrm{C} 14$ & $119.9(2)$ & $\mathrm{C} 246-\mathrm{C} 241-\mathrm{C} 24$ & $120.8(2)$ \\
\hline $\mathrm{C} 141-\mathrm{C} 142-\mathrm{C} 143$ & $120.2(2)$ & $\mathrm{C} 243-\mathrm{C} 242-\mathrm{C} 241$ & $120.2(2)$ \\
\hline $\mathrm{C} 141-\mathrm{C} 142-\mathrm{H} 142$ & 119.9 & $\mathrm{C} 243-\mathrm{C} 242-\mathrm{H} 242$ & 119.9 \\
\hline $\mathrm{C} 143-\mathrm{C} 142-\mathrm{H} 142$ & 119.9 & $\mathrm{C} 241-\mathrm{C} 242-\mathrm{H} 242$ & 119.9 \\
\hline $\mathrm{C} 142-\mathrm{C} 143-\mathrm{C} 144$ & $120.2(2)$ & $\mathrm{C} 244-\mathrm{C} 243-\mathrm{C} 242$ & $120.9(2)$ \\
\hline $\mathrm{C} 142-\mathrm{C} 143-\mathrm{H} 143$ & 119.9 & $\mathrm{C} 244-\mathrm{C} 243-\mathrm{H} 243$ & 119.6 \\
\hline $\mathrm{C} 144-\mathrm{C} 143-\mathrm{H} 143$ & 119.9 & $\mathrm{C} 242-\mathrm{C} 243-\mathrm{H} 243$ & 119.6 \\
\hline $\mathrm{C} 145-\mathrm{C} 144-\mathrm{C} 143$ & $119.7(2)$ & $\mathrm{C} 243-\mathrm{C} 244-\mathrm{C} 245$ & $119.5(2)$ \\
\hline C145-C144-H144 & 120.1 & $\mathrm{C} 243-\mathrm{C} 244-\mathrm{H} 244$ & 120.3 \\
\hline $\mathrm{C} 143-\mathrm{C} 144-\mathrm{H} 144$ & 120.1 & $\mathrm{C} 245-\mathrm{C} 244-\mathrm{H} 244$ & 120.3 \\
\hline $\mathrm{C} 144-\mathrm{C} 145-\mathrm{C} 146$ & $119.8(2)$ & $\mathrm{C} 244-\mathrm{C} 245-\mathrm{C} 246$ & $120.1(2)$ \\
\hline $\mathrm{C} 144-\mathrm{C} 145-\mathrm{H} 145$ & 120.1 & $\mathrm{C} 244-\mathrm{C} 245-\mathrm{H} 245$ & 120.0 \\
\hline $\mathrm{C} 146-\mathrm{C} 145-\mathrm{H} 145$ & 120.1 & $\mathrm{C} 246-\mathrm{C} 245-\mathrm{H} 245$ & 120.0 \\
\hline $\mathrm{C} 145-\mathrm{C} 146-\mathrm{C} 141$ & $121.0(2)$ & $\mathrm{C} 245-\mathrm{C} 246-\mathrm{C} 241$ & $120.6(2)$ \\
\hline $\mathrm{C} 145-\mathrm{C} 146-\mathrm{H} 146$ & 119.5 & $\mathrm{C} 245-\mathrm{C} 246-\mathrm{H} 246$ & 119.7 \\
\hline $\mathrm{C} 141-\mathrm{C} 146-\mathrm{H} 146$ & 119.5 & $\mathrm{C} 241-\mathrm{C} 246-\mathrm{H} 246$ & 119.7 \\
\hline $\mathrm{C} 14-\mathrm{C} 15-\mathrm{N} 11$ & $105.72(19)$ & $\mathrm{C} 24-\mathrm{C} 25-\mathrm{N} 21$ & $105.88(19)$ \\
\hline $\mathrm{C} 14-\mathrm{C} 15-\mathrm{C} 151$ & $128.9(2)$ & $\mathrm{C} 24-\mathrm{C} 25-\mathrm{C} 251$ & $128.6(2)$ \\
\hline $\mathrm{N} 11-\mathrm{C} 15-\mathrm{C} 151$ & $125.36(19)$ & $\mathrm{N} 21-\mathrm{C} 25-\mathrm{C} 251$ & $125.49(18)$ \\
\hline $\mathrm{C} 152-\mathrm{C} 151-\mathrm{C} 156$ & $118.9(2)$ & $\mathrm{C} 252-\mathrm{C} 251-\mathrm{C} 256$ & $118.8(2)$ \\
\hline $\mathrm{C} 152-\mathrm{C} 151-\mathrm{C} 15$ & $121.9(2)$ & $\mathrm{C} 252-\mathrm{C} 251-\mathrm{C} 25$ & $118.9(2)$ \\
\hline $\mathrm{C} 156-\mathrm{C} 151-\mathrm{C} 15$ & $119.0(2)$ & $\mathrm{C} 256-\mathrm{C} 251-\mathrm{C} 25$ & $122.0(2)$ \\
\hline $\mathrm{C} 151-\mathrm{C} 152-\mathrm{C} 153$ & $120.2(2)$ & $\mathrm{C} 253-\mathrm{C} 252-\mathrm{C} 251$ & $121.0(2)$ \\
\hline $\mathrm{C} 151-\mathrm{C} 152-\mathrm{H} 152$ & 119.9 & $\mathrm{C} 253-\mathrm{C} 252-\mathrm{H} 252$ & 119.5 \\
\hline $\mathrm{C} 153-\mathrm{C} 152-\mathrm{H} 152$ & 119.9 & $\mathrm{C} 251-\mathrm{C} 252-\mathrm{H} 252$ & 119.5 \\
\hline $\mathrm{C} 154-\mathrm{C} 153-\mathrm{C} 152$ & $120.5(2)$ & $\mathrm{C} 252-\mathrm{C} 253-\mathrm{C} 254$ & $119.9(2)$ \\
\hline $\mathrm{C} 154-\mathrm{C} 153-\mathrm{H} 153$ & 119.8 & $\mathrm{C} 252-\mathrm{C} 253-\mathrm{H} 253$ & 120.1 \\
\hline $\mathrm{C} 152-\mathrm{C} 153-\mathrm{H} 153$ & 119.7 & $\mathrm{C} 254-\mathrm{C} 253-\mathrm{H} 253$ & 120.1 \\
\hline $\mathrm{C} 153-\mathrm{C} 154-\mathrm{C} 155$ & $119.7(2)$ & $\mathrm{C} 255-\mathrm{C} 254-\mathrm{C} 253$ & $119.6(2)$ \\
\hline $\mathrm{C} 153-\mathrm{C} 154-\mathrm{H} 154$ & 120.2 & $\mathrm{C} 255-\mathrm{C} 254-\mathrm{H} 254$ & 120.2 \\
\hline $\mathrm{C} 155-\mathrm{C} 154-\mathrm{H} 154$ & 120.2 & $\mathrm{C} 253-\mathrm{C} 254-\mathrm{H} 254$ & 120.2 \\
\hline $\mathrm{C} 154-\mathrm{C} 155-\mathrm{C} 156$ & $120.1(2)$ & $\mathrm{C} 254-\mathrm{C} 255-\mathrm{C} 256$ & $120.6(2)$ \\
\hline C154-C155-H155 & 119.9 & $\mathrm{C} 254-\mathrm{C} 255-\mathrm{H} 255$ & 119.7 \\
\hline $\mathrm{C} 156-\mathrm{C} 155-\mathrm{H} 155$ & 119.9 & $\mathrm{C} 256-\mathrm{C} 255-\mathrm{H} 255$ & 119.7 \\
\hline $\mathrm{C} 151-\mathrm{C} 156-\mathrm{C} 155$ & $120.6(2)$ & $\mathrm{C} 255-\mathrm{C} 256-\mathrm{C} 251$ & $120.1(2)$ \\
\hline $\mathrm{C} 151-\mathrm{C} 156-\mathrm{H} 156$ & 119.7 & $\mathrm{C} 255-\mathrm{C} 256-\mathrm{H} 256$ & 120.0 \\
\hline $\mathrm{C} 155-\mathrm{C} 156-\mathrm{H} 156$ & 119.7 & $\mathrm{C} 251-\mathrm{C} 256-\mathrm{H} 256$ & 120.0 \\
\hline $\mathrm{C} 12-\mathrm{N} 11-\mathrm{C} 111-\mathrm{C} 112$ & $-107.9(2)$ & $\mathrm{C} 22-\mathrm{N} 21-\mathrm{C} 211-\mathrm{C} 212$ & $-110.1(2)$ \\
\hline $\mathrm{C} 15-\mathrm{N} 11-\mathrm{C} 111-\mathrm{C} 112$ & $74.2(2)$ & $\mathrm{C} 25-\mathrm{N} 21-\mathrm{C} 211-\mathrm{C} 212$ & $74.3(3)$ \\
\hline $\mathrm{N} 11-\mathrm{C} 111-\mathrm{C} 112-\mathrm{O} 112$ & $64.7(2)$ & $\mathrm{N} 21-\mathrm{C} 211-\mathrm{C} 212-\mathrm{O} 212$ & $66.0(2)$ \\
\hline $\mathrm{N} 11-\mathrm{C} 111-\mathrm{C} 112-\mathrm{C} 113$ & $-173.05(19)$ & $\mathrm{N} 21-\mathrm{C} 211-\mathrm{C} 212-\mathrm{C} 213$ & $-171.0(2)$ \\
\hline $\mathrm{C} 15-\mathrm{N} 11-\mathrm{C} 12-\mathrm{N} 13$ & $0.4(2)$ & $\mathrm{C} 25-\mathrm{N} 21-\mathrm{C} 22-\mathrm{N} 23$ & $0.6(2)$ \\
\hline $\mathrm{C} 111-\mathrm{N} 11-\mathrm{C} 12-\mathrm{N} 13$ & $-177.80(18)$ & $\mathrm{C} 211-\mathrm{N} 21-\mathrm{C} 22-\mathrm{N} 23$ & $-175.67(19)$ \\
\hline
\end{tabular}




$\begin{array}{ll}\text { C15-N11-C12-C121 } & 177.1(2) \\ \text { C111-N11-C12-C121 } & -1.1(3) \\ \text { N13-C12-C121-C122 } & -138.1(2) \\ \text { N11-C12-C121-C122 } & 45.5(3) \\ \text { N13-C12-C121-C126 } & 39.2(3) \\ \text { N11-C12-C121-C126 } & -137.1(2) \\ \text { C126-C121-C122-C123 } & 1.0(3) \\ \text { C12-C121-C122-C123 } & 178.3(2) \\ \text { C121-C122-C123-C124 } & 1.2(4) \\ \text { C122-C123-C124-C125 } & -1.7(4) \\ \text { C122-C123-C124-N124 } & 177.6(2) \\ \text { C123-C124-N124-O12 } & 11.7(4) \\ \text { C125-C124-N124-O12 } & -169.0(3) \\ \text { C123-C124-N124-O13 } & -166.8(3) \\ \text { C125-C124-N124-O13 } & 12.5(4) \\ \text { C123-C124-C125-C126 } & -0.2(4) \\ \text { N124-C124-C125-C126 } & -179.5(2) \\ \text { C124-C125-C126-C121 } & 2.4(4) \\ \text { C122-C121-C126-C125 } & -2.9(4) \\ \text { C12-C121-C126-C125 } & 179.6(2) \\ \text { N11-C12-N13-C14 } & -0.3(2) \\ \text { C121-C12-N13-C14 } & -177.24(19) \\ \text { C12-N13-C14-C15 } & 0.0(2) \\ \text { C12-N13-C14-C141 } & 177.4(2) \\ \text { N13-C14-C141-C142 } & 46.6(3) \\ \text { C15-C14-C141-C142 } & -136.5(2) \\ \text { N13-C14-C141-C146 } & -133.2(2) \\ \text { C15-C14-C141-C146 } & 43.6(3) \\ \text { C146-C141-C142-C143 } & -0.9(3) \\ \text { C14-C141-C142-C143 } & 179.3(2) \\ \text { C141-C142-C143-C144 } & 0.7(4) \\ \text { C142-C143-C144-C145 } & 0.1(4) \\ \text { C143-C144-C145-C146 } & -0.7(4) \\ \text { C144-C145-C146-C141 } & 0.5(4) \\ \text { C142-C141-C146-C145 } & 0.3(3) \\ \text { C14-C141-C146-C145 } & -179.9(2) \\ \text { N13-C14-C15-N11 } & 0.2(2) \\ \text { C141-C14-C15-N11 } & -177.0(2) \\ \text { N13-C14-C15-C151 } & -179.5(2) \\ \text { C141-C14-C15-C151 } & 3.4(4) \\ \text { C12-N11-C15-C14 } & -0.4(2) \\ \text { C111-N11-C15-C14 } & 177.92(18) \\ \text { C12-N11-C15-C151 } & 179.31(19) \\ \text { C111-N11-C15-C151 } & -2.4(3) \\ \text { C14-C15-C151-C152 } & -120.2(3) \\ \text { N11-C15-C151-C152 } & 60.2(3) \\ \text { C14-C15-C151-C156 } & 54.2(3) \\ \text { N11-C15-C151-C156 } & -125.4(2) \\ & \end{array}$

\begin{tabular}{|c|c|}
\hline $\mathrm{C} 25-\mathrm{N} 21-\mathrm{C} 22-\mathrm{C} 221$ & $-178.8(2)$ \\
\hline $\mathrm{C} 211-\mathrm{N} 21-\mathrm{C} 22-\mathrm{C} 221$ & $4.9(3)$ \\
\hline $\mathrm{N} 23-\mathrm{C} 22-\mathrm{C} 221-\mathrm{C} 226$ & $-137.8(2)$ \\
\hline $\mathrm{N} 21-\mathrm{C} 22-\mathrm{C} 221-\mathrm{C} 226$ & $41.5(3)$ \\
\hline $\mathrm{N} 23-\mathrm{C} 22-\mathrm{C} 221-\mathrm{C} 222$ & $36.9(3)$ \\
\hline $\mathrm{N} 21-\mathrm{C} 22-\mathrm{C} 221-\mathrm{C} 222$ & $-143.8(2)$ \\
\hline $\mathrm{C} 226-\mathrm{C} 221-\mathrm{C} 222-\mathrm{C} 223$ & $0.4(3)$ \\
\hline $\mathrm{C} 22-\mathrm{C} 221-\mathrm{C} 222-\mathrm{C} 223$ & $-174.5(2)$ \\
\hline $\mathrm{C} 221-\mathrm{C} 222-\mathrm{C} 223-\mathrm{C} 224$ & $0.7(3)$ \\
\hline $\mathrm{C} 222-\mathrm{C} 223-\mathrm{C} 224-\mathrm{C} 225$ & $-0.9(4)$ \\
\hline $\mathrm{C} 222-\mathrm{C} 223-\mathrm{C} 224-\mathrm{N} 224$ & $177.6(2)$ \\
\hline $\mathrm{C} 223-\mathrm{C} 224-\mathrm{N} 224-\mathrm{O} 23$ & $-168.0(2)$ \\
\hline $\mathrm{C} 225-\mathrm{C} 224-\mathrm{N} 224-\mathrm{O} 23$ & $10.5(3)$ \\
\hline $\mathrm{C} 223-\mathrm{C} 224-\mathrm{N} 224-\mathrm{O} 22$ & $12.1(3)$ \\
\hline $\mathrm{C} 225-\mathrm{C} 224-\mathrm{N} 224-\mathrm{O} 22$ & $-169.3(2)$ \\
\hline $\mathrm{C} 223-\mathrm{C} 224-\mathrm{C} 225-\mathrm{C} 226$ & $0.0(4)$ \\
\hline $\mathrm{N} 224-\mathrm{C} 224-\mathrm{C} 225-\mathrm{C} 226$ & $-178.5(2)$ \\
\hline $\mathrm{C} 224-\mathrm{C} 225-\mathrm{C} 226-\mathrm{C} 221$ & $1.2(3)$ \\
\hline $\mathrm{C} 222-\mathrm{C} 221-\mathrm{C} 226-\mathrm{C} 225$ & $-1.4(3)$ \\
\hline $\mathrm{C} 22-\mathrm{C} 221-\mathrm{C} 226-\mathrm{C} 225$ & $173.1(2)$ \\
\hline $\mathrm{N} 21-\mathrm{C} 22-\mathrm{N} 23-\mathrm{C} 24$ & $-0.2(2)$ \\
\hline $\mathrm{C} 221-\mathrm{C} 22-\mathrm{N} 23-\mathrm{C} 24$ & $179.24(19)$ \\
\hline $\mathrm{C} 22-\mathrm{N} 23-\mathrm{C} 24-\mathrm{C} 25$ & $-0.3(2)$ \\
\hline $\mathrm{C} 22-\mathrm{N} 23-\mathrm{C} 24-\mathrm{C} 241$ & $179.2(2)$ \\
\hline $\mathrm{N} 23-\mathrm{C} 24-\mathrm{C} 241-\mathrm{C} 242$ & $34.5(3)$ \\
\hline $\mathrm{C} 25-\mathrm{C} 24-\mathrm{C} 241-\mathrm{C} 242$ & $-146.2(2)$ \\
\hline $\mathrm{N} 23-\mathrm{C} 24-\mathrm{C} 241-\mathrm{C} 246$ & $-144.8(2)$ \\
\hline $\mathrm{C} 25-\mathrm{C} 24-\mathrm{C} 241-\mathrm{C} 246$ & $34.5(3)$ \\
\hline $\mathrm{C} 246-\mathrm{C} 241-\mathrm{C} 242-\mathrm{C} 243$ & $-0.5(3)$ \\
\hline $\mathrm{C} 24-\mathrm{C} 241-\mathrm{C} 242-\mathrm{C} 243$ & $-179.7(2)$ \\
\hline $\mathrm{C} 241-\mathrm{C} 242-\mathrm{C} 243-\mathrm{C} 244$ & $0.1(3)$ \\
\hline $\mathrm{C} 242-\mathrm{C} 243-\mathrm{C} 244-\mathrm{C} 245$ & $0.0(4)$ \\
\hline $\mathrm{C} 243-\mathrm{C} 244-\mathrm{C} 245-\mathrm{C} 246$ & $0.1(4)$ \\
\hline $\mathrm{C} 244-\mathrm{C} 245-\mathrm{C} 246-\mathrm{C} 241$ & $-0.4(4)$ \\
\hline $\mathrm{C} 242-\mathrm{C} 241-\mathrm{C} 246-\mathrm{C} 245$ & $0.6(3)$ \\
\hline $\mathrm{C} 24-\mathrm{C} 241-\mathrm{C} 246-\mathrm{C} 245$ & $179.9(2)$ \\
\hline $\mathrm{N} 23-\mathrm{C} 24-\mathrm{C} 25-\mathrm{N} 21$ & $0.6(2)$ \\
\hline $\mathrm{C} 241-\mathrm{C} 24-\mathrm{C} 25-\mathrm{N} 21$ & $-178.8(2)$ \\
\hline $\mathrm{N} 23-\mathrm{C} 24-\mathrm{C} 25-\mathrm{C} 251$ & $-176.2(2)$ \\
\hline $\mathrm{C} 241-\mathrm{C} 24-\mathrm{C} 25-\mathrm{C} 251$ & $4.4(4)$ \\
\hline $\mathrm{C} 22-\mathrm{N} 21-\mathrm{C} 25-\mathrm{C} 24$ & $-0.7(2)$ \\
\hline $\mathrm{C} 211-\mathrm{N} 21-\mathrm{C} 25-\mathrm{C} 24$ & $175.74(19)$ \\
\hline $\mathrm{C} 22-\mathrm{N} 21-\mathrm{C} 25-\mathrm{C} 251$ & $176.2(2)$ \\
\hline $\mathrm{C} 211-\mathrm{N} 21-\mathrm{C} 25-\mathrm{C} 251$ & $-7.3(3)$ \\
\hline $\mathrm{C} 24-\mathrm{C} 25-\mathrm{C} 251-\mathrm{C} 252$ & $56.1(3)$ \\
\hline $\mathrm{N} 21-\mathrm{C} 25-\mathrm{C} 251-\mathrm{C} 252$ & $-120.1(2)$ \\
\hline $\mathrm{C} 24-\mathrm{C} 25-\mathrm{C} 251-\mathrm{C} 256$ & $-118.7(3)$ \\
\hline $\mathrm{N} 21-\mathrm{C} 25-\mathrm{C} 251-\mathrm{C} 256$ & $65.1(3)$ \\
\hline
\end{tabular}




$\begin{array}{llll}\mathrm{C} 156-\mathrm{C} 151-\mathrm{C} 152-\mathrm{C} 153 & 0.9(3) & \mathrm{C} 256-\mathrm{C} 251-\mathrm{C} 252-\mathrm{C} 253 & 0.6(3) \\ \mathrm{C} 15-\mathrm{C} 151-\mathrm{C} 152-\mathrm{C} 153 & 175.3(2) & \mathrm{C} 25-\mathrm{C} 251-\mathrm{C} 252-\mathrm{C} 253 & -174.4(2) \\ \mathrm{C} 151-\mathrm{C} 152-\mathrm{C} 153-\mathrm{C} 154 & -1.2(3) & \mathrm{C} 251-\mathrm{C} 252-\mathrm{C} 253-\mathrm{C} 254 & -0.1(4) \\ \mathrm{C} 152-\mathrm{C} 153-\mathrm{C} 154-\mathrm{C} 155 & 0.7(4) & \mathrm{C} 252-\mathrm{C} 253-\mathrm{C} 254-\mathrm{C} 255 & -0.6(4) \\ \mathrm{C} 153-\mathrm{C} 154-\mathrm{C} 155-\mathrm{C} 156 & 0.2(4) & \mathrm{C} 253-\mathrm{C} 254-\mathrm{C} 255-\mathrm{C} 256 & 0.9(3) \\ \mathrm{C} 152-\mathrm{C} 151-\mathrm{C} 156-\mathrm{C} 155 & 0.0(3) & \mathrm{C} 254-\mathrm{C} 255-\mathrm{C} 256-\mathrm{C} 251 & -0.4(3) \\ \mathrm{C} 15-\mathrm{C} 151-\mathrm{C} 156-\mathrm{C} 155 & -174.6(2) & \mathrm{C} 252-\mathrm{C} 251-\mathrm{C} 256-\mathrm{C} 255 & -0.3(3) \\ \mathrm{C} 154-\mathrm{C} 155-\mathrm{C} 156-\mathrm{C} 151 & -0.5(4) & \mathrm{C} 25-\mathrm{C} 251-\mathrm{C} 256-\mathrm{C} 255 & 174.50(19)\end{array}$

Hydrogen-bond geometry $\left(A,{ }^{\circ}\right)$

$\mathrm{Cg} 1$ and $\mathrm{Cg} 5$ are the centroids of the $\mathrm{N} 11 / \mathrm{C} 12 / \mathrm{N} 13 / \mathrm{C} 14 / \mathrm{C} 15$ and $\mathrm{N} 21 / \mathrm{C} 22 / \mathrm{N} 23 / \mathrm{C} 24 / \mathrm{C} 25$ rings, respectively.

\begin{tabular}{lllll}
\hline$D-\mathrm{H} \cdots A$ & $D-\mathrm{H}$ & $\mathrm{H} \cdots A$ & $D \cdots A$ & $D-\mathrm{H} \cdots A$ \\
\hline $\mathrm{O} 212-\mathrm{H} 21 O \cdots \mathrm{N} 13$ & $0.89(4)$ & $1.90(4)$ & $2.773(2)$ & $168(3)$ \\
$\mathrm{C} 253-\mathrm{H} 253 \cdots \mathrm{O} 13$ & 0.95 & 2.69 & $3.491(4)$ & 143 \\
$\mathrm{O} 112-\mathrm{H} 11 O \cdots \mathrm{N} 23^{\mathrm{i}}$ & $0.88(4)$ & $1.94(4)$ & $2.798(2)$ & $165(3)$ \\
$\mathrm{C} 155-\mathrm{H} 155 \cdots \mathrm{O} 22^{\mathrm{i}}$ & 0.95 & 2.57 & $3.244(3)$ & 128 \\
$\mathrm{C} 152-\mathrm{H} 152 \cdots \mathrm{O} 212^{\mathrm{ii}}$ & 0.95 & 2.66 & $3.263(3)$ & 122 \\
$\mathrm{C} 153-\mathrm{H} 153 \cdots \mathrm{N} 21^{\mathrm{ii}}$ & 0.95 & 2.74 & $3.682(3)$ & 170 \\
$\mathrm{C} 243-\mathrm{H} 243 \cdots \mathrm{O} 23^{\mathrm{iii}}$ & 0.95 & 2.59 & $3.542(4)$ & 176 \\
$\mathrm{C} 242-\mathrm{H} 242 \cdots \mathrm{O} 112^{\mathrm{iv}}$ & 0.95 & 2.71 & $3.338(3)$ & 124 \\
$\mathrm{C} 256-\mathrm{H} 256 \cdots \mathrm{O} 112^{\mathrm{v}}$ & 0.95 & 2.57 & $3.208(3)$ & 125 \\
$\mathrm{C} 145-\mathrm{H} 145 \cdots \mathrm{O} 22^{\mathrm{vi}}$ & 0.95 & 2.58 & $3.439(3)$ & 151 \\
$\mathrm{C} 153-\mathrm{H} 153 \cdots C g 5^{\mathrm{ii}}$ & 0.95 & 2.61 & $3.469(2)$ & 151 \\
$\mathrm{C} 255-\mathrm{H} 255 \cdots C g 1^{\mathrm{v}}$ & 0.95 & 2.66 & $3.544(3)$ & 154 \\
\hline
\end{tabular}

Symmetry codes: (i) $x, y-1, z$; (ii) $-x+1,-y+1,-z+1$; (iii) $x-1, y, z$; (iv) $x, y+1, z$; (v) $-x+1,-y+1,-z+2$; (vi) $-x+2,-y+2,-z+1$. 